\title{
Simultaneous visualization of the flow inside and around droplets generated in microchannels
}

\section{Journal Article}

\section{Author(s):}

Duxenneuner, Manuela R.; Fischer, Peter; Windhab, Erich J.; Cooper-White, Justin J.

Publication date:

2014-04

Permanent link:

https://doi.org/10.3929/ethz-b-000071685

Rights / license:

In Copyright - Non-Commercial Use Permitted

\section{Originally published in:}

Microfluidics and Nanofluidics 16(4), https://doi.org/10.1007/s10404-013-1259-9 


\title{
Simulation of rockfall trajectories with consideration of rock shape
}

\author{
R.I. Leine • A. Schweizer • M. Christen • J. Glover • \\ P. Bartelt • W. Gerber
}

Received: 28 January 2013 / Accepted: 19 July 2013 / Published online: 7 September 2013

(C) Springer Science+Business Media Dordrecht 2013

\begin{abstract}
The aim of the paper is to develop a fully 3D simulation technique for rockfall dynamics taking rock shape into account and using the state-of-the-art methods of multibody dynamics and nonsmooth contact dynamics. The rockfall simulation technique is based on the nonsmooth contact dynamics method with hard contact laws. The rock is modeled as an arbitrary convex polyhedron and the terrain model is based on a high resolution digital elevation model. A specialized friction law for rockfall is proposed which allows for the description of scarring behavior (i.e., rocks tend to slide over the terrain before lift-off). The influence of rock geometry on rockfall dynamics is studied through two well-chosen numerical simulations.
\end{abstract}

Keywords Natural hazards · Nonsmooth dynamics - Unilateral constraint - Contact . Friction

\section{Introduction}

Rockfall is a serious natural hazard in mountainous areas and represents a major threat to infrastructure, transportation lines, and people (Fig. 1). The identification of potential rockfall

R.I. Leine $(\varangle) \cdot$ A. Schweizer

Institute of Mechanical Systems, Department of Mechanical and Process Engineering, ETH Zurich, 8092 Zurich, Switzerland

e-mail: remco.leine@imes.mavt.ethz.ch

M. Christen · J. Glover · P. Bartelt

WSL Institute for Snow and Avalanche Research SLF, Flüelastrasse 11, 7260 Davos Dorf, Switzerland

P. Bartelt

e-mail: bartelt@slf.ch

W. Gerber

Swiss Federal Institute for Forest, Snow and Landscape Research WSL, Zürcherstrasse 111, 8903 Birmensdorf, Switzerland

e-mail: werner.gerber@wsl.ch 
Fig. 1 Rockfall incident at the highway A2 near Gurtnellen, Switzerland, on 31 May 2006 leading to two casualties (photo Swiss Federal Roads Office)

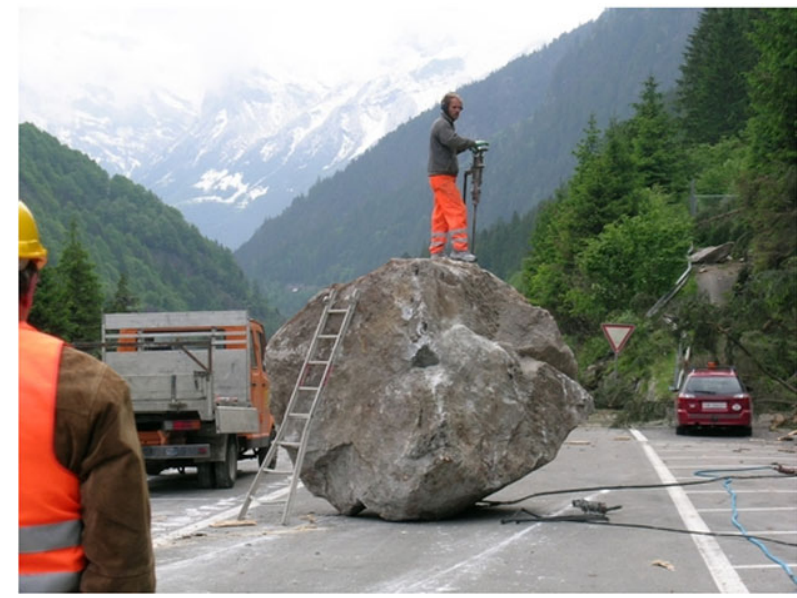

starting zones, the calculation of the rock trajectories in complex three-dimensional terrain, and the interaction of falling rocks with protection measures, such as rockfall dams, catching nets, and mountain forests, are three major components of the rockfall problem and research. These problems have become especially urgent, as warming trends in world climate are having a significant impact on mountain permafrost, destabilizing rockfall starting zones [27]. Recent events have demonstrated that rockfall is also a dangerous secondary hazard after extreme earthquakes.

Methods to predict rockfall trajectories in complex three-dimensional terrain (given an unstable source area) have great practical value as they can be used to determine the danger of rockfall run-out zones as well as dimension protection measures. However, the simulation of a falling rock is extremely challenging because when a rock moves downslope, various modes of motion are possible: free fall, sliding, rolling, and bouncing with slip or stick. The run-out distance, lateral spreading and jump heights are strongly influenced by the shape of the rock and the interaction with the terrain and other obstacles such as trees.

The present work is concerned with the development of a fully three-dimensional rigidbody rockfall trajectory model that accounts for rock shape and, therefore, provides a more accurate and physically consistent description of the rock-terrain interaction. For practical usage, it is necessary to establish a computationally reliable model that employs constitutive parameters, which characterize terrain surface properties such as hardness, scree content, soil cover, and vegetation independent of rock size and shape. This would place rockfall modeling on a solid physical foundation and allow the widespread application of trajectory models for risk assessment and the design of mitigation measures.

The rock shape is in this paper considered to be a convex polyhedral, being a good approximation of the angular rocks relevant for rockfall. The rocks are released by fracturing from the bedrock and are therefore edged by nature as opposed to rounded glacial boulders. Moreover, the polyhedral shape of rocks can originate from the rock morphology. For instance, columnar basalt rocks are prismatic with a predominantly hexagonal cross-section, and are therefore tall polyhedrons. The typically polyhedral shape of the rocks has a large influence on their dynamics.

The aim of the present paper is to develop rockfall simulation techniques, which take the rock shape into account using the state-of-the-art methods of multibody dynamics and nonsmooth contact dynamics. The influence of rock geometry on rockfall dynamics is studied in this paper through two well-chosen numerical simulations. 
We begin with a short overview in Sect. 2 of how rock shape is treated in existing rockfall trajectory modeling approaches, thereby placing our work in the context of current rockfall modeling practice. Subsequently, we start with the introduction of a fully three-dimensional rigid-body rockfall trajectory model. In Sect. 3, the geometric and mechanical aspects of the model of the rock, the terrain and their interaction are discussed. Section 4 is concerned with the numerical time-integration of the equations of motion. Two simulations are documented in Sect. 5 and the paper closes with conclusions in Sect. 6.

\section{Overview of existing rockfall modeling approaches}

Following the review article of Volkwein et al. [42], we distinguish between four different types of rockfall simulation codes: what one may call a "horizontal" 2D approach, a "vertical" 2D approach, the so-called 2.5D approach (being a concatenation of the latter two) and $3 \mathrm{D}$ simulation approaches. The goal of all these approaches is to compute the space curve $(x(t), y(t), z(t))$ of the center of mass, i.e., the trajectory of the rock. The most simple "horizontal" $2 \mathrm{D}$ approach computes the $(x(t), y(t))$ motion assuming that the rock is a "sliding block" on the terrain, whereas the $z$ coordinate is defined by the height of the terrain. A "vertical" 2D approach simulates the trajectory $(s(t), z(t))$ with bounces and flight phases on a slope profile along a predefined path $(x(s), y(s))$ on the map with path parameter $s$, e.g., along the line of steepest descent. A concatenation of these two $2 \mathrm{D}$ approaches leads to the $2.5 \mathrm{D}$ approach, which first computes the $(x, y)$ motion using a horizontal 2D approach, thereby defining the path of a slope profile, and subsequently performs a vertical $2 \mathrm{D}$ simulation on the previously computed slope profile. Finally, 3D approaches directly simulate the spatial translational motion $(x(t), y(t), z(t))$ but not necessarily the rotational motion of the rock. Although $2 \mathrm{D}$ and $2.5 \mathrm{D}$ rockfall simulation techniques have predominantly been used in the past, a gradual shift can be observed toward 3D rockfall simulation. An extensive overview of rockfall simulation codes is given in [42]. Here, we will only briefly discuss the most modern 3D rockfall simulation codes and their properties. Three-dimensional rockfall simulation codes may be classified according to the geometric modeling of the boulder:

1. Point-mass models: A boulder is considered to be a point-mass, i.e., a point like particle. The only kinetic property of the boulder is its mass. The kinematics of the boulder is described by a three-dimensional position vector and a translational velocity vector. Sometimes the point-mass is equipped with a geometry, which is only used to calculate the contact with the ground or obstacles (typically trees).

2. Sphere models: A boulder is considered to be a rigid homogeneous sphere with a certain radius. The kinetic properties of the boulder are its mass and a moment of inertia, which does not depend on the axis of rotation. The kinematics of the boulder is described by a position vector, whereas the orientation of the boulder is irrelevant. The generalized velocity is described by a translational velocity vector and an angular velocity vector.

3. Rigid complex shape models: A boulder is endowed with a geometry, which is parameterized by a number of geometric parameters. For example, a boulder may be a rectangular block with three parameters (height, width, depth) or be an arbitrary polyhedron. The properties of the boulder are its mass, the location of the center of mass within the body and the inertia tensor. The kinematics of the boulder is described by a position vector and a singularity-free description of the orientation (e.g., by using a quaternion). The generalized velocity is described by a translational velocity vector and an angular velocity vector. 
4. Discrete element complex shape models: A boulder is considered to be a collection of spheres or point-mass particles together with elastic interconnecting elements. Hence, also the deformation of the boulder is to some extent taken into account.

Point-mass models neither take into account the geometry of the rock nor the energy distribution between angular and translational degrees of freedom. The modeling of boulders with spheres leads to excessive run-out lengths because a sphere will keep on rolling on an inclined slope and may only stop moving at a local minimum in the landscape. Moreover, gyroscopic forces are absent in a sphere model. Complex shape models do not have these discrepancies, but ask for much more computational effort and user specification of rock shape. The full three-dimensional simulation of rockfall with complex shape models allows to study the influence of rock geometry on the run-out lengths and the scattering of the trajectories. Rockfall research has been dominated in the past by simpler point mass and 2D models because of their computational efficiency, which is needed in Monte Carlo simulations. Although more computational demanding, 3D rockfall simulation with complex shape models is now becoming competitive due to fast and cheap personal computers (less than one second computation time per simulated trajectory on an ordinary PC is typical).

Various modeling approaches exist for the simulation of mechanical bodies, of which the finite element method, the distinct element method and the rigid-body approach are the most important ones. A FEM approach allows predicting stresses and deformations. The distinct element method considers a body to be a collection of (spherical) particles with (visco-)elastic connecting elements. The rigid-body approach neglects deformations of the bodies and assumes that the deformations, which are due to applied and contact forces, are much smaller than the macroscopic motion of the bodies. Hence, if only the macroscopic motion of the contacting objects is of interest, as is the case in rockfall simulation, then the rigid-body approach is numerically more efficient and most natural. Most rockfall simulation packages therefore use the rigid-body approach.

Finally, simulation codes for rigid/elastic bodies with contact (to which rockfall simulation codes belong) can be classified according to the modeling of the contact interaction (rock-terrain interaction):

1. Rebound impact models: Point-mass models are always combined with an empirical rebound model with normal and tangential rebound factors. Stochastic approaches have been proposed to account for the variability of the rebound.

2. Penalty approach and regularization techniques: The rationale behind these techniques is that all forces are described by single-valued force laws, which are a function of the generalized positions and velocities. The penalty approach models the interaction in normal direction with a one-sided spring or spring-dashpot (Kelvin-Voigt) element. It therefore allows for a (small) interpenetration of the contacting bodies and the normal contact force is proportional to this interpenetration. The tangential contact force, which is due to dry friction, is usually assumed to obey Coulomb's friction law. The friction characteristic is regularized (e.g., with an arctangent function) such that the friction force is a single-valued function of the relative velocity.

3. Hard contact laws/Nonsmooth contact dynamics method: Hard contact laws use setvalued force laws to describe the interaction between contacting bodies. The impenetrability of contacting bodies (rock and terrain) is therefore described exactly by using an inequality complementarity (Signorini's law). Furthermore, Coulomb's law of dry friction is employed in its unregularized form. Stiction of the rock on the terrain can therefore be described. Apart from force laws for the contact force, also impact laws need to be specified. 
The penalty method is widely used in the contact dynamics community due to its simplicity, e.g., [17, 23, 25, 29]. All forces are described by single-valued force laws and the penalty/regularization approach therefore leads to an equation of motion in the form of an ordinary (but stiff) differential equation for which standard solvers are available. It is sometimes argued that the penalty approach is more realistic than a hard contact law because it can (in a crude sense) describe the elasticity of the contacting partners in the same way as Hertz contact law does [29]. The stiffness parameter used in the penalty approach is, however, almost always chosen much smaller than the Hertzian contact stiffness in order to avoid numerical integration problems, which result from the stiff differential equation. The stiffness parameter of the penalty approach can therefore not be regarded as a physical (or modeling) parameter but has to be regarded as a numerical parameter. Usually, a one-sided springdashpot element is used to model the restitution behavior. The amount of restitution can be steered with the damping coefficient of the spring-dashpot element. Unfortunately, the penalty stiffness also influences the impact process and restitution (see [6], Sect. 2.1.3). Numerical parameters are therefore mixed with modeling parameters in the penalty approach which is a significant drawback. Moreover, a straightforward regularization of Coulomb's friction law leads to another cumbersome effect: The friction force vanishes for zero relative velocity and stiction is no longer possible. This means that an object can never stand still on an inclined slope, because the friction force is not able to make equilibrium with the gravitational force. Hence, a rock with a regularized friction law will always (slowly) slide down the terrain, contrary to what is observed in reality. More elaborate regularized friction models have been developed which can describe stiction (LuGre model [7], Karnopp model [26], switch model [34]), using internal states or switching techniques, but these methods are not suitable for the simulation of multibody systems with many frictional unilateral constraints.

The nonsmooth contact dynamics method [1, 6, 14, 18, 24, 33, 35, 37] uses hard contact laws. The impenetrability of the contacts in the "hard" modeling approach asks for set-valued force laws (Signorini's law, Coulomb's friction law) and instantaneous impacts laws (of Newton or Poisson-type), which give at collision times an instantaneous change in the velocities of all contacting bodies. The formulation of unilateral and frictional contact behavior therefore requires constitutive contact laws, which are set-valued. The equations of motion are therefore no longer described by ordinary differential equations but by measure differential inclusions. Numerical time-integration methods measure differential inclusions are continually being developed and improved [1, 41].

With the above classifications in mind, we will give a brief overview of the existing 3D rockfall simulation codes. The majority of the existing 3D rockfall simulation packages use point-mass models. Examples are STONE [22] and its successor HY-STONE [10] as well as RockFall Analyst [28] and PICUS-ROCKnROLL [43]. These point-mass models are always combined with an empirical rebound model with normal and tangential rebound factors.

RockyFor3D [5], which has been developed since 1998 [12], uses essentially a sphere model in combination with a stochastic impact model which leads to a lateral dispersion of the trajectories. RockyFor3D has the possibility to use rectangular, ellipsoidal and spherical boulder forms as input for the simulations, but this shape information is only used to calculate the moments of inertia and only a spherical shape is used to calculate the contact with the terrain or trees.

CRSP-3D (Colorado Rockfall Simulation Program) [3] employs the distinct element method together with a penalty approach. Nonspherical boulder forms such as a rectangular cuboid or a cylinder are approximated by a collection of rigid spheres with interconnecting elastic elements. 
The rockfall simulation code STAR3-D [11, 30, 31], which is being developed at LCPC (Paris) and IRSTEA (formerly known as CEMAGREF, Grenoble), is a fully threedimensional rockfall simulation code with a rigid complex shape model. However, STAR3$\mathrm{D}$ is limited to parallelepiped rock shapes. The nonsmooth contact dynamics method in combination with the percussion law of Frémond [14] is used in STAR3-D to model the rockterrain interaction and the rock-tree interaction. As the numerical integration method has not been documented, one may assume that the standard Moreau time-stepping method [37] has been employed.

The code LMGC90 [13, 24, 37] is a general purpose open-source software developed at the LMGC laboratory (Montpellier), capable of modeling a large collection of deformable or undeformable particles of various shapes, with various interaction laws (e.g., hard contacts). LMGC90 is used in [39] to simulate rockfall in quarries and galleries. However, LMGC90 does not have a specialized user-interface for rockfall simulation in mountainous areas (i.e., GIS-based elevation model for the terrain, forest interaction, etc.).

Having discussed the various ways to classify rockfall simulation approaches and existing simulation software packages, we start presenting the simulation techniques used and developed in this paper.

\section{The simulation model}

The simulation method proposed in this paper has the following characteristics:

1. a full 3D simulation model for rockfall based on the nonsmooth contact dynamics method with hard contact laws,

2. a complex shape model which consists of an arbitrary convex polyhedron and is therefore not restricted to blocks (or parallelepiped shapes),

3. a terrain model which is based on a high resolution digital elevation model,

4. a specialized friction law for rockfall is proposed in this paper which allows for the description of scarring behavior (i.e., rocks tend to slide over the terrain before lift-off),

5. a drag force model for forest interaction,

6. an energetically consistent numerical time-integration technique, which guarantees that there is no energy increase during flight phases.

The proposed simulation method is implemented in the software package RAMMS (Rapid Mass MovementS), being a simulation tool for the prediction of natural hazards such as avalanches and debris flow developed at the WSL Institute for Snow and Avalanche Research SLF [8, 9]. The software RAMMS has an extensive graphical user-interface and a high resolution digital elevation model (topographical satellite data or airborne LIDAR) is used as input data. The integration of the simulation code in an existing software package for natural hazards is a prerequisite for the use of the tool by a larger community (geophysicists, geologists, engineers).

The rockfall simulation model presented here considers the rock to be a perfectly rigid and unbreakable three-dimensional body in the form of a convex polyhedron. It may come into contact with the impenetrable surface of the terrain. First, we will introduce various coordinate frames in Sect. 3.1 and define the terrain geometry in Sect. 3.2. Subsequently, the rock geometry, kinematics, and dynamics are extensively described in the remaining part of this section. The formulation of standard contact and impact laws from nonsmooth dynamics will briefly be described in Sect. 3.6. However, some phenomena of rockfall dynamics cannot be described by standard contact laws. Therefore, a slippage dependent friction coefficient model is introduced in Sect. 3.7. 
Fig. 2 The terrain model resides in the inertial frame $I$ with origin $O$. The geometry of the rock is specified in the body-fixed frame $B$ with origin $C$. The eigenframe $K$ with the center of mass $S$ as its origin is used in the simulation

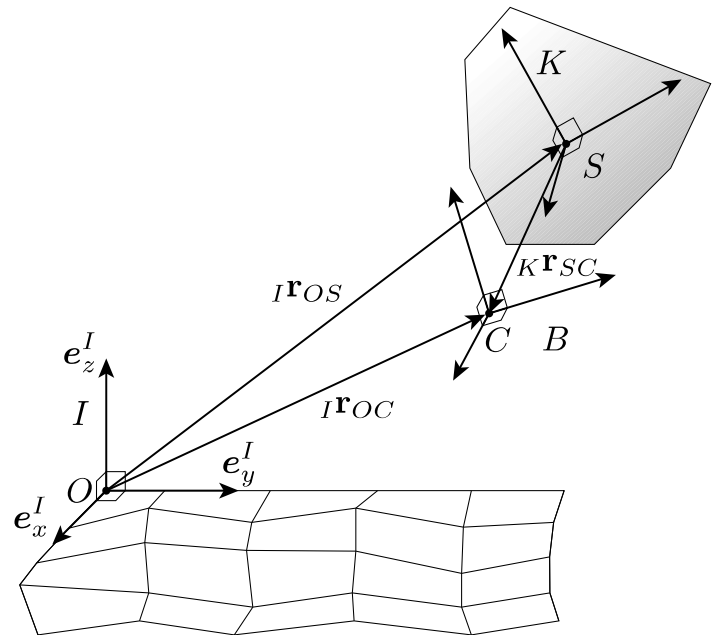

\subsection{Coordinate systems}

In the following, the three orthonormal coordinate frames $I, K$, and $B$ are used, as shown in Fig. 2. The frame $I$ with origin $O$ is the inertial frame in which the terrain geometry is specified. The rock resides in a body-fixed coordinate frame $K$, which is translated and rotated with respect to $I$ by a time-dependent transformation. The frame $K$ is defined as the eigenframe of the rock's inertia tensor and is attached at the center of mass $S$ of the rock. Initially, the geometry of the rock may be specified in a body fixed coordinate frame $B$ with origin $C$, which does not agree with frame $K$, but is translated and rotated with respect to $K$ by a constant transformation.

The rotation of frame $K$ with respect to frame $I$ is represented by a unit quaternion $\boldsymbol{p}_{I K}=\left(e_{0} \boldsymbol{e}\right)^{\mathrm{T}}$. The quaternion coordinate $e_{0}$ stands for the "real part" of the quaternion $\boldsymbol{p}_{I K}$ and the three-dimensional vector $\boldsymbol{e}=\left(e_{1}, e_{2}, e_{3}\right)$ for the "imaginary part." The coordinate transformation ${ }_{K} \boldsymbol{r} \mapsto{ }_{I} \boldsymbol{r}$ of the coordinates of $\boldsymbol{r}$ in the $K$ frame to the $I$ frame is given by the coordinate transformation matrix

$$
\boldsymbol{A}_{I K}=\left(\begin{array}{ccc}
\left(e_{0}^{2}+e_{1}^{2}-e_{2}^{2}-e_{3}^{2}\right) & 2\left(e_{1} e_{2}-e_{0} e_{3}\right) & 2\left(e_{0} e_{2}+e_{1} e_{3}\right) \\
2\left(e_{0} e_{3}+e_{1} e_{2}\right) & \left(e_{0}^{2}-e_{1}^{2}+e_{2}^{2}-e_{3}^{2}\right) & 2\left(e_{2} e_{3}-e_{0} e_{1}\right) \\
2\left(e_{1} e_{3}-e_{0} e_{2}\right) & 2\left(e_{0} e_{1}+e_{2} e_{3}\right) & \left(e_{0}^{2}-e_{1}^{2}-e_{2}^{2}+e_{3}^{2}\right)
\end{array}\right)
$$

such that ${ }_{I} \boldsymbol{r}=\boldsymbol{A}_{I K K} \boldsymbol{r}$.

\subsection{Terrain geometry}

A high resolution digital elevation model, giving the height of the terrain on a fixed grid, is used to define the terrain geometry. The height values $h_{k, l}$ of the digital elevation model at the vertices indexed by $k \in\left\{0,1, \ldots, n_{x}\right\}$ and $l \in\left\{0, \ldots, n_{y}\right\}$ are specified on a rectangular uniform grid with cell-sizes $l_{x}, l_{y}$, and $n_{x}, n_{y}$ cells in $x$-resp. $y$-direction. The height values $h_{k, l}$ are bilinearly interpolated providing a height map $z:(x, y) \mapsto z(x, y)$.

A specific location $(x, y)$ is located in the grid-cell $(k, l)=\left(\left\lfloor x / l_{x}\right\rfloor,\left\lfloor y / l_{y}\right\rfloor\right)$, where $\lfloor\cdot\rfloor$ denotes the floor function, and has the interpolation parameters $(\eta, \xi) \in[0,1]^{2}$, where

$$
\eta=x / l_{x}-k, \quad \xi=y / l_{y}-l .
$$


Fig. 3 The contact frame at a point $Q$ on the terrain is spanned by the normal vector $\boldsymbol{n}$ and the two tangential vectors $t_{1}, t_{2}$

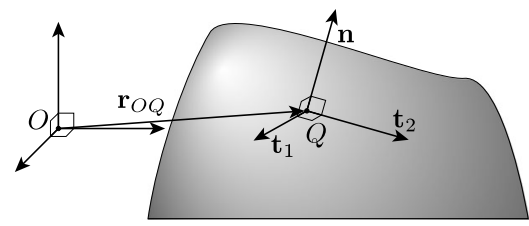

The interpolation parameters $(\eta, \xi)$ are fed into four weight functions $w_{i, j}:[0,1]^{2} \rightarrow[0,1]$

$$
\begin{aligned}
& w_{0,0}(\eta, \xi)=(1-\eta)(1-\xi), \quad w_{1,1}(\eta, \xi)=\eta \xi \\
& w_{0,1}(\eta, \xi)=(1-\eta) \xi, \quad w_{1,0}(\eta, \xi)=\eta(1-\xi),
\end{aligned}
$$

which weigh the height values of the four corners of the corresponding grid cell. The interpolated height $z(x, y)$ is given by the weighted sum

$$
z(x, y)=z_{k l}(\eta, \xi)=\sum_{i, j=0}^{1,1} h_{k+i, l+j} w_{i, j}(\eta, \xi),
$$

where $(k, l)$ and $(\eta, \xi)$ are functions of $(x, y)$. The corresponding point $Q$ on the terrain is represented as a vector with respect to the inertial frame $I$ with origin $O$

$$
{ }_{I} \boldsymbol{r}_{O Q, k l}(\eta, \xi)=\left(\begin{array}{c}
(\eta+k) l_{x} \\
(\xi+l) l_{y} \\
z_{k l}(\eta, \xi)
\end{array}\right)
$$

Modeling parameters which are considered to be position dependent, such as the friction and restitution coefficients, are interpolated in the same manner on the same grid.

Each point $(x, y, z)$ on the terrain is endowed with a local contact frame $\left(\boldsymbol{n}, \boldsymbol{t}_{1}, \boldsymbol{t}_{2}\right)$; see Fig. 3. The contact frame defines the contact plane and its normal. It therefore describes the directions of the normal and tangential components of the contact force as well as the kinematic contact quantities (relative velocities in normal and tangential directions). Force laws for each of the components of the contact force are given in Sect. 3.6. The normal direction $\boldsymbol{n}$ of the contact frame determines the direction of the normal force, which prevents the rock from falling through the terrain. The two tangential directions, $\boldsymbol{t}_{1}$ and $\boldsymbol{t}_{2}$, span the contact plane in which the friction force lives. The normal can be obtained by building the cross product

$$
\boldsymbol{n}_{k l}(\eta, \xi)=\partial_{\eta I} \boldsymbol{r}_{O Q, k l}(\eta, \xi) \times \partial_{\xi I} \boldsymbol{r}_{O Q, k l}(\eta, \xi)=\left(\begin{array}{c}
-\partial_{\eta} z_{k l}(\eta, \xi) l_{y} \\
-\partial_{\xi} z_{k l}(\eta, \xi) l_{x} \\
l_{x} l_{y}
\end{array}\right)
$$

After evaluating the directional derivatives of $z_{k l}$ and simplifying the resulting expressions the normal becomes

$$
\boldsymbol{n}_{k l}(\eta, \xi)=\left(\begin{array}{c}
-\left(\left(h_{k+1, l}-h_{k, l}\right)(1-\xi)+\left(h_{k+1, l+1}-h_{k, l+1}\right) \xi\right) l_{y} \\
-\left(\left(h_{k, l+1}-h_{k, l}\right)(1-\eta)+\left(h_{k+1, l+1}-h_{k+1, l}\right) \eta\right) l_{x} \\
l_{x} l_{y}
\end{array}\right)
$$




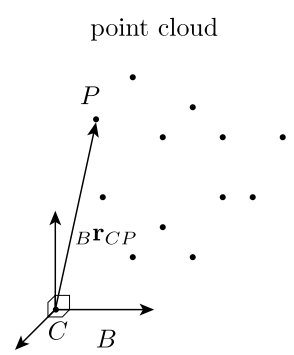

a)

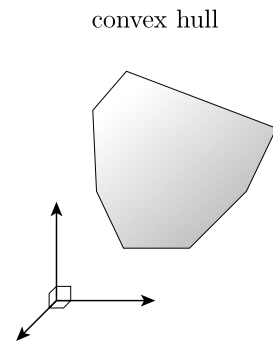

b)

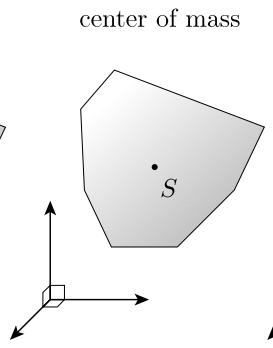

c) eigenframe

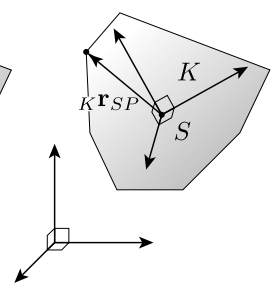

d)

Fig. 4 Generation of the rock geometry and inertia properties

Two additional vectors $\boldsymbol{t}_{1}, \boldsymbol{t}_{2}$ are needed to span the plane orthogonal to $\boldsymbol{n}$. The rotation of $\boldsymbol{t}_{1}$ and $\boldsymbol{t}_{2}$ around the normal is arbitrary and a suitable choice has to be made, e.g.,

$$
\boldsymbol{e}=\underset{\boldsymbol{v} \in\left\{\boldsymbol{e}_{x}^{I}, \boldsymbol{e}_{y}^{I}, \boldsymbol{e}_{z}^{I}\right\}}{\operatorname{argmin}}|\boldsymbol{v} \cdot \boldsymbol{n}|, \quad \boldsymbol{t}_{1}=\boldsymbol{e} \times \boldsymbol{n}, \quad \boldsymbol{t}_{2}=\boldsymbol{n} \times \boldsymbol{t}_{1} .
$$

\subsection{The rock model}

The rock geometry is considered to be a three-dimensional convex polyhedron and can therefore be defined as the convex hull of a finite point set in the three-dimensional space. Real rocks, which are found in the field, are being digitized by the WSL Institute for Snow and Avalanche Research SLF using laser scanning techniques. In this way, a cloud of points is generated (typically consisting of a few hundred points), which is subsequently converted to a convex polyhedron, and used in the simulation model. The generation of the rock geometry is illustrated in Fig. 4. The rock geometry is specified by a point cloud in the body-fixed coordinate frame $B$ with the origin $C$ (Fig. 4(a)). The convex hull of the point cloud is constructed after which the center of mass $S$ and the inertia tensor of the rock are determined under the assumption of a homogeneous mass distribution (Fig. 4(b) and 4(c)). Lastly, the eigenframe $K$ of the inertia tensor is calculated and chosen as the new body-fixed frame with origin $S$ (Fig. 4(d)).

Several forces act on the rock and determine its trajectory as shown in Fig. 5. The force

$$
{ }_{I} \boldsymbol{F}_{g}=-m g_{I} \boldsymbol{e}_{z}^{I}
$$

models the gravitational force, where $m$ is the mass of the rock and $g$ is the gravitational acceleration, which acts in the negative $z$ direction of the inertial frame $I$. The presence of forest and bush can have a significant influence on the rock trajectory. In the absence of sophisticated models for vegetation interaction, we propose a drag force ${ }_{I} \boldsymbol{F}_{d}$ as being a very crude approximation for the influence of forest and bush on the rock trajectory. This drag force is assumed to be linear in the velocity ${ }_{I} \boldsymbol{v}_{S}$ of the center of mass $S$ and acts in the opposite direction of the velocity

$$
{ }_{I} \boldsymbol{F}_{d}=-c_{d}\left({ }_{I} \boldsymbol{r}_{O S}\right)_{I} \boldsymbol{v}_{S},
$$

where the drag coefficient

$$
c_{d}\left({ }_{I} \boldsymbol{r}_{O S}\right)= \begin{cases}\hat{c}_{d}(x, y) & \text { if } z<z_{\text {height }} \\ 0 & \text { otherwise }\end{cases}
$$


Fig. 5 External forces acting on the rock are the gravitational force ${ }_{I} \boldsymbol{F}_{g}$ and frictional contact forces ${ }_{I} \boldsymbol{F}_{c}$. For a rock with its center of mass lying inside the drag force layer with height $z_{\text {height }}$, the drag force ${ }_{I} \boldsymbol{F}_{d}$ is enabled and acts in the opposite direction of the center of mass velocity ${ }_{I} \boldsymbol{v}_{S}$ of the rock

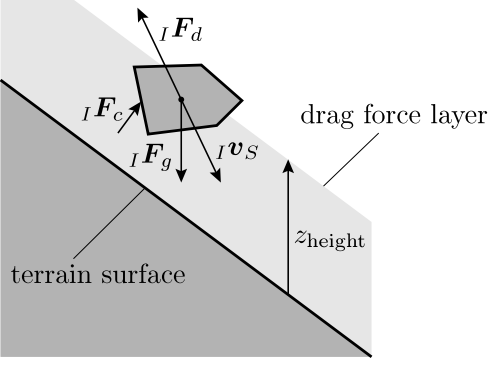

varies depending on the current location ${ }_{I} \boldsymbol{r}_{O S}=(x, y, z)$ of the center of mass of the rock and is only enabled below a certain height $z_{\text {height }}$ above the terrain as shown in Fig. 5. In this way, the effect of tree height is taken into account.

Additionally, frictional contact forces ${ }_{I} \boldsymbol{F}_{c}$ model the interaction of the rock with the terrain. In Fig. 5, the corresponding arrow is not attached at the center of mass to suggest that these forces act at arbitrary material points. Depending on the current configuration of the rock, the number of active contact forces varies. Each contact force acts at a different material point on the surface of the rock and their introduction into the equations of motion is therefore more involved (see Sect. 3.4).

\subsection{Equations of motion and impact equation}

In this section, the equations of motion and the impact equations of the rock are discussed. The rock is assumed to be a three-dimensional rigid body, with three translational and three rotational degrees of freedom. Its configuration at time $t$ is represented by the tuple of generalized coordinates

$$
\boldsymbol{q}:=\left(\begin{array}{c}
{ }_{I} \boldsymbol{r}_{O S} \\
\boldsymbol{p}_{I K}
\end{array}\right) \in \mathbb{R}^{7}, \quad\left\|\boldsymbol{p}_{I K}\right\|=1
$$

where ${ }_{I} \boldsymbol{r}_{O S}$ represents the coordinates of the position of the body's center of mass $S$ relative to the origin $O$ of the inertial frame and $\boldsymbol{p}_{I K}=\left(e_{0} \boldsymbol{e}\right)^{\mathrm{T}}$ are the coordinates of the unit quaternion (hence the normalization condition) representing the orientation of the bodyfixed coordinate frame $K$ with respect to the inertial frame $I$.

The time-derivative $\dot{\boldsymbol{q}}$ of the generalized coordinates (12) is represented by a reduced vector of generalized velocities

$$
\boldsymbol{u}:=\left(\begin{array}{c}
{ }_{I} \boldsymbol{v}_{S} \\
{ }_{K} \boldsymbol{\Omega}
\end{array}\right) \in \mathbb{R}^{6},
$$

where ${ }_{I} \boldsymbol{v}_{S}$ is the translational velocity of the center of mass represented in the inertial frame $I$ and ${ }_{K} \boldsymbol{\Omega}$ is the angular velocity represented in the body-fixed frame $K$. The time derivative of the generalized coordinates is related to the generalized velocities (13) through the linear map (see [38])

$$
\dot{\boldsymbol{q}}=\boldsymbol{F}\left(\boldsymbol{p}_{I K}\right) \boldsymbol{u} \text { a.e., } \quad \boldsymbol{F}\left(\boldsymbol{p}_{I K}\right)=\left(\begin{array}{cc}
\boldsymbol{I}_{3 \times 3} & 0 \\
0 & -\frac{1}{2} \boldsymbol{e}^{\mathrm{T}} \\
0 & \frac{1}{2} e_{0} \boldsymbol{I}+\frac{1}{2} \widetilde{\boldsymbol{e}}
\end{array}\right),
$$


where $\boldsymbol{I}_{3 \times 3}$ is a $3 \times 3$ unit matrix and ${ }^{\sim}$ denotes the linear operator which turns a threedimensional vector into a $3 \times 3$ skew-symmetric matrix such that $\tilde{\boldsymbol{a}} \boldsymbol{b}=\boldsymbol{a} \times \boldsymbol{b}$.

We first consider the rock in free flight (and deal with the contact forces later). The time evolution of the translational velocity ${ }_{I} \boldsymbol{v}_{S}$ is described by the linear momentum equation

$$
m_{I} \dot{\boldsymbol{v}}_{S}={ }_{I} \boldsymbol{F}_{d}+{ }_{I} \boldsymbol{F}_{g}
$$

where the right-hand side is composed of the drag force ${ }_{I} \boldsymbol{F}_{d}$; see (10), and the gravitational force ${ }_{I} \boldsymbol{F}_{g}$, see (9). The time evolution of the angular velocity ${ }_{K} \boldsymbol{\Omega}$ is given by the spin equation

$$
{ }_{K} \boldsymbol{\Theta}_{S K} \dot{\boldsymbol{\Omega}}+{ }_{K} \boldsymbol{\Omega} \times{ }_{K} \boldsymbol{\Theta}_{S K} \boldsymbol{\Omega}={ }_{K} \boldsymbol{M}^{a},
$$

where ${ }_{K} \boldsymbol{\Theta}_{S}$ is the inertia tensor represented in the body-fixed $K$ frame and ${ }_{K} \boldsymbol{M}^{a}$ contains external free torques. However, ${ }_{K} \boldsymbol{M}^{a}=\mathbf{0}$ as the only external moments result from contact forces. The benefit of choosing a representation of the spin equation in the body-fixed frame $K$ is that the inertia tensor $\boldsymbol{\Theta}_{S}$ is constant and diagonal in frame $K$ and can be expressed as

$$
{ }_{K} \boldsymbol{\Theta}_{S}=\operatorname{diag}\left(\Theta_{1}, \Theta_{2}, \Theta_{3}\right)
$$

where $\Theta_{1}, \Theta_{2}, \Theta_{3} \in \mathbb{R}$ are the principal moments of inertia.

The linear momentum equation (15) and the spin equation (16) are combined into the equations of motion

$$
\boldsymbol{M} \dot{\boldsymbol{u}}-\boldsymbol{h}(\boldsymbol{q}, \boldsymbol{u})=\mathbf{0}
$$

with the constant and diagonal mass matrix $\boldsymbol{M}$ and the force term $\boldsymbol{h}(\boldsymbol{q}, \boldsymbol{u})$, which contains the gyroscopic terms and external finite forces, given by

$$
\boldsymbol{M}=\left(\begin{array}{cc}
m \boldsymbol{I}_{3 \times 3} & \mathbf{0}_{3 \times 3} \\
\boldsymbol{0}_{3 \times 3} & { }_{K} \boldsymbol{\Theta}_{S}
\end{array}\right), \quad \boldsymbol{h}(\boldsymbol{q}, \boldsymbol{u})=\left(\begin{array}{c}
{ }_{I} \boldsymbol{F}_{d}+{ }_{I} \boldsymbol{F}_{g} \\
-{ }_{K} \boldsymbol{\Omega} \times{ }_{K} \boldsymbol{\Theta}_{S K} \boldsymbol{\Omega}
\end{array}\right)
$$

Subsequently, we account for the contributions of unilateral constraint forces and friction forces and augment the equations of motion (18) with Lagrangian multipliers

$$
\boldsymbol{M} \dot{\boldsymbol{u}}-\boldsymbol{h}(\boldsymbol{q}, \boldsymbol{u})=\boldsymbol{W}(\boldsymbol{q}) \lambda \text { a.e. }
$$

where $\boldsymbol{W}$ is the matrix of generalized force directions and $\lambda$ is a vector containing all the contact forces. The contact forces ${ }_{I} \boldsymbol{F}_{c} \in \mathbb{R}^{3}$, which have been introduced Sect. 3.3, appear in the equations of motion (18) through the generalized forces $\boldsymbol{W}(\boldsymbol{q}) \boldsymbol{\lambda}$. The equations of motion (20) are fulfilled almost everywhere except at impact time instants $t_{i}$ for which velocity jumps occur due to impulsive contact forces. At these time instants, the velocities $\boldsymbol{u}(t)$ and accelerations $\dot{\boldsymbol{u}}(t)$ are undefined and the differential equations have therefore to be complemented by the impact equations

$$
\boldsymbol{M}\left(\boldsymbol{u}^{+}\left(t_{i}\right)-\boldsymbol{u}^{-}\left(t_{i}\right)\right)=\boldsymbol{W}\left(\boldsymbol{q}\left(t_{i}\right)\right) \boldsymbol{\Lambda}\left(t_{i}\right),
$$

which relate the left- and right-limits of the velocities $\boldsymbol{u}^{-}$resp. $\boldsymbol{u}^{+}$to the impulsive contact forces $\boldsymbol{\Lambda}$. The gyroscopic terms and the finite external force terms are nonimpulsive and do not have a contribution in the impact equation. 
Fig. 6 Collision detection for three different rock geometry points $P_{1}, P_{2}, P_{3}$. For the points $P_{1}, P_{2}$, the gap function has a negative sign because they lie below the terrain surface $\mathcal{S}$

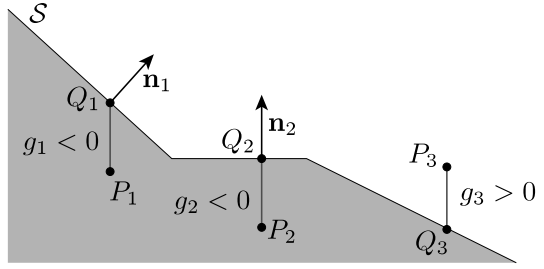

By introducing the differential measures $\mathrm{d} \boldsymbol{u}=\dot{\boldsymbol{u}} \mathrm{d} t+\left(\boldsymbol{u}^{+}-\boldsymbol{u}^{-}\right) \mathrm{d} \eta$ and $\mathrm{d} \boldsymbol{P}=\boldsymbol{\lambda} \mathrm{d} t+\boldsymbol{\Lambda} \mathrm{d} \eta$, where $\mathrm{d} t$ is the Lebesgue measure and $\mathrm{d} \eta$ is the Dirac measure the differential equations of motion and the impact equations are combined into the measure differential equation

$$
\boldsymbol{M} \mathrm{d} \boldsymbol{u}-\boldsymbol{h}(\boldsymbol{q}, \boldsymbol{u}, t) \mathrm{d} t-\boldsymbol{W}(\boldsymbol{q}) \mathrm{d} \boldsymbol{P}=\mathbf{0},
$$

also called equality of measures [35]. The differential measure of percussions $\mathrm{d} \boldsymbol{P}$ accounts for contributions of frictional contact forces and frictional impulsive contact forces. The differential measure formulation (22) can be directly discretized, which leads to a dedicated time-integration scheme for multibody systems with impulsive motion (see Sect. 4).

\subsection{Contact detection and contact forces}

Whenever the rock touches the terrain, the manifold of contact is represented as a discrete collection of contact points. Each contact point introduces normal and tangential contact forces into the equations of motion. Assuming that the resolution of the terrain is coarser than that of the rock geometry, only the points of the rock geometry are tested against penetration with the terrain to determine whether a contact is potentially active (on displacement level) or not. This information is obtained by looking at the sign of the gap function

$$
g_{N}\left(x_{P}, y_{P}, z_{P}\right)=z_{P}-z\left(x_{P}, y_{P}\right)
$$

of each of the geometry points $P$, where $x_{P}, y_{P}, z_{P}$ represent the inertial coordinates of $P$ and $z\left(x_{P}, y_{P}\right)$ is the height of the terrain below $P$. This operation corresponds to projecting the point $P$ vertically onto the terrain and comparing the height of the projected point $Q$ with the height of the original point $P$; see Fig. 6. If $g_{N}>0$, then the point $P$ is above the terrain and, therefore, higher than its vertical projection point $Q$. If $g_{N}=0$, then the point $P$ is on the terrain and agrees with its projection $Q$. If $g_{N}<0$, then the point $P$ is below the terrain and there is a certain amount of penetration between the rock and the terrain.

For numerical reasons, we have to allow for a very small amount of penetration, which is only needed in a Boolean sense: When $g_{N} \leq 0$, there is contact; when $g_{N}>0$ there is no contact. Whenever $g_{N} \leq 0$ holds, contact forces, acting on the point $P$ of the rock, are computed which obey a contact law. Loosely speaking, the contact law ensures that no further penetration occurs, or that the contact is lost $\left(g_{N}>0\right)$.

Consider a contact pair $(P, Q)$ and the contact frame $C=\left({ }_{I} \boldsymbol{n},{ }_{I} \boldsymbol{t}_{1},{ }_{I} \boldsymbol{t}_{2}\right)$ attached to $Q$. Contact between the rock and the terrain $\left(g_{N} \leq 0\right)$ invokes a contact force $\lambda=$ $\left(\lambda_{N}, \lambda_{T 1}, \lambda_{T 2}\right)^{\mathrm{T}}$ in the three basis directions of the contact frame $C$. The normal contact force $\lambda_{N}$ guarantees the unilaterality of the contact, whereas $\lambda_{T 1}$ and $\lambda_{T 2}$ are the tangential components of the contact force due to Coulomb friction. The contact force $\lambda$ has a generalized force $f_{C}=\boldsymbol{W} \boldsymbol{\lambda}$ in the equation of motion, where $\boldsymbol{W}$ is the matrix of generalized force directions. 
The generalized force directions $\boldsymbol{W}$ of a force-element can be obtained from its kinematic relations as a consequence of the principle of virtual work, or, equivalently, the principle of virtual power, e.g. see [18]. Hereto, let $\gamma_{C}=\left(\gamma_{N}, \gamma_{T 1}, \gamma_{T 2}\right)^{\mathrm{T}}$ denote the contact velocity, being the relative velocity of point $P$ with respect to point $Q$ in the three basis directions of the contact frame $C$ :

$$
\boldsymbol{\gamma}_{C}={ }_{C} \boldsymbol{v}_{P}-{ }_{C} \boldsymbol{v}_{Q}
$$

Using $\boldsymbol{v}_{Q}=\mathbf{0}$, as the terrain does not move, together with the coordinate transformation matrix $\boldsymbol{A}_{I C}=\boldsymbol{A}_{C I}^{\mathrm{T}}=\left({ }_{I} \boldsymbol{n}_{I} \boldsymbol{t}_{1}{ }_{I} \boldsymbol{t}_{2}\right)$ we obtain the contact velocity $\boldsymbol{\gamma}_{C}=\boldsymbol{A}_{C I I} \boldsymbol{v}_{P}=\boldsymbol{A}_{I C I}^{\mathrm{T}} \boldsymbol{v}_{P}$, which can be expanded using the rigid body formula $\boldsymbol{v}_{P}=\boldsymbol{v}_{S}+\boldsymbol{\Omega} \times \boldsymbol{r}_{S P}$ giving

$$
\begin{aligned}
\boldsymbol{\gamma}_{C} & =\boldsymbol{A}_{I C}^{\mathrm{T}}\left({ }_{I} \boldsymbol{v}_{S}+{ }_{I} \boldsymbol{\Omega} \times{ }_{I} \boldsymbol{r}_{S P}\right) \\
& =\boldsymbol{A}_{I C}^{\mathrm{T}}\left({ }_{I} \boldsymbol{v}_{S}+\boldsymbol{A}_{I K}{ }_{K} \boldsymbol{\Omega} \times{ }_{K} \boldsymbol{r}_{S P}\right),
\end{aligned}
$$

where ${ }_{I} \boldsymbol{v}_{S}$ is the translational velocity of the center of mass represented in the inertial frame $I$ and ${ }_{K} \boldsymbol{\Omega}$ the angular velocity represented in the body-fixed frame $K$. The position vector from the center of mass $S$ to point $P$ is given by ${ }_{K} \boldsymbol{r}_{S P}$, represented in the $K$ frame. Using the antisymmetry of the cross-product, i.e., $\boldsymbol{a} \times \boldsymbol{b}=-\boldsymbol{b} \times \boldsymbol{a}=-\widetilde{\boldsymbol{b}} \boldsymbol{a}$ where $\tilde{\boldsymbol{b}}$ is a skewsymmetric matrix, one obtains

$$
\begin{aligned}
\boldsymbol{\gamma}_{C} & =\boldsymbol{A}_{I C}^{\mathrm{T}}\left({ }_{I} \boldsymbol{v}_{S}-\boldsymbol{A}_{I K{ }_{K}} \tilde{\boldsymbol{r}}_{S P K} \boldsymbol{\Omega}\right) \\
& =\boldsymbol{A}_{I C}^{\mathrm{T}}\left[\begin{array}{ll}
\boldsymbol{I}_{3 \times 3} & -\boldsymbol{A}_{I K}{ }_{K} \widetilde{\boldsymbol{r}}_{S P}
\end{array}\right] \underbrace{\left(\begin{array}{l}
{ }_{I} \boldsymbol{v}_{S} \\
{ }_{K} \boldsymbol{\Omega}
\end{array}\right)}_{\boldsymbol{u}}
\end{aligned}
$$

in which the generalized velocities $\boldsymbol{u}$ have been separated. Finally, we introduce the generalized force directions as

$$
\boldsymbol{W}(\boldsymbol{q})=\left[\begin{array}{ll}
\boldsymbol{I}_{3 \times 3} & -\boldsymbol{A}_{I K}{ }_{K} \widetilde{\boldsymbol{r}}_{S P}
\end{array}\right]^{\mathrm{T}} \boldsymbol{A}_{I C},
$$

being dependent on the generalized position $\boldsymbol{q}$, and write the contact velocity as

$$
\boldsymbol{\gamma}_{C}=\boldsymbol{W}^{\mathrm{T}} \boldsymbol{u}
$$

which is a linear form in the generalized velocity $\boldsymbol{u}$. Using the principle of virtual power, for which the velocities are varied and the positions are kept fixed during the variation process, we equate the virtual power of the contact force $\lambda$ to the virtual power of the generalized contact force $f_{C}$, such that

$$
\delta \boldsymbol{\gamma}_{C}^{\mathrm{T}} \boldsymbol{\lambda}=\delta \boldsymbol{u}^{\mathrm{T}} \boldsymbol{f}_{C},
$$

where $\delta \boldsymbol{u}$ and $\delta \boldsymbol{\gamma}_{C}$ are the virtual generalized and contact velocity, respectively. Substitution of the kinematic relationship $\delta \boldsymbol{\gamma}_{C}=\boldsymbol{W}^{\mathrm{T}} \delta \boldsymbol{u}$ (28) in the virtual power equality (29) yields

$$
\delta \boldsymbol{u}^{\mathrm{T}} \boldsymbol{W} \boldsymbol{\lambda}=\delta \boldsymbol{u}^{\mathrm{T}} \boldsymbol{f}_{C} .
$$

The latter has to hold for all virtual velocities $\delta \boldsymbol{u}$ whereupon we infer that the generalized contact force is given by $\boldsymbol{f}_{C}=\boldsymbol{W} \boldsymbol{\lambda}$. 


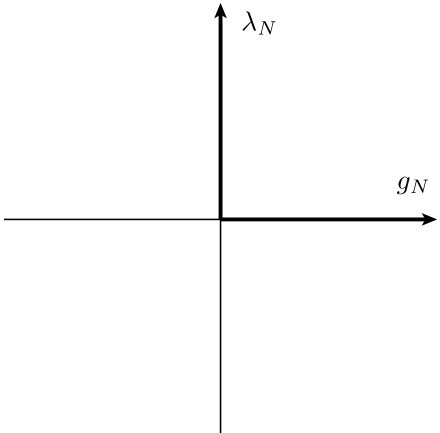

(a)

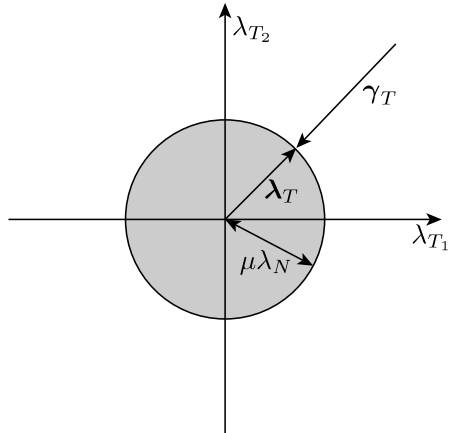

(b)

Fig. 7 Normal contact law (a) and Coulomb friction law (b)

For every potentially active contact $i$, an additional Lagrange multiplier term $\boldsymbol{W}_{i} \boldsymbol{\lambda}_{i}$ is introduced into the equations of motion. The three-dimensional contact force vector

$$
\lambda_{i}=\left(\begin{array}{c}
\lambda_{N_{i}} \\
\lambda_{T_{i}}
\end{array}\right) \in \mathbb{R}^{3}
$$

contains the normal force $\lambda_{N i} \in \mathbb{R}$ and the friction force $\lambda_{T i}=\left(\lambda_{T 1 i} \lambda_{T 2 i}\right) \in \mathbb{R}^{2}$.

\subsection{Contact laws}

The constitutive behavior of the contact is described by various contact laws for the contact forces as well as impact laws for impulsive contact forces.

\subsubsection{Normal contact force law}

The contact law in normal direction is given by the inequality complementarity

$$
\lambda_{N} \geq 0 \wedge g_{N} \geq 0 \wedge \lambda_{N} g_{N}=0,
$$

being a set-valued relationship between the gap function $g_{N}$ and the normal contact force $\lambda_{N}$ (Fig. 7(a)). Physically, the inequality complementarity conditions (32), often referred to as the Signorini conditions, express that either the normal force is nonnegative $\lambda_{N} \geq 0$ and the contact is closed $g_{N}=0$, or the contact is open $g_{N}>0$ and the force has to vanish $\lambda_{N}=0$. The relations (32) are often written in the more compact and elegant form

$$
0 \leq \lambda_{N} \perp g_{N} \geq 0,
$$

which is equivalent to (32). Nonsmooth dynamics uses the concept of normal cone inclusions to formulate inequality complementarities or more general set-valued force laws $[18,35]$. In particular, the inequality complementarity (32) is expressed by

$$
-g_{N} \in \mathcal{N}_{\mathbb{R}_{0}^{+}}\left(\lambda_{N}\right),
$$

where $\mathcal{N}_{\mathbb{R}_{0}^{+}}\left(\lambda_{N}\right)$ denotes the normal cone on the set $\mathbb{R}_{0}^{+}$of all nonnegative real numbers, i.e.,

$$
\mathcal{N}_{\mathbb{R}_{0}^{+}}\left(\lambda_{N}\right)=\left\{\begin{array}{ll}
\{0\} & \text { for } \lambda_{N}>0 \\
(-\infty, 0] & \lambda_{N}=0
\end{array} .\right.
$$


For the numerical implementation, it is necessary to formulate the normal contact law, which is originally stated on position level, by a set-valued force law on velocity level [18]

$$
\begin{array}{ll}
g_{N}>0: & \lambda_{N}=0, \\
g_{N}=0: & -\gamma_{N} \in \mathcal{N}_{\mathbb{R}_{0}^{+}}\left(\lambda_{N}\right) .
\end{array}
$$

\subsubsection{Coulomb friction law}

The tangential contact force $\lambda_{T}$ is assumed to obey spatial Coulomb's friction law. For the isotropic Coulomb's friction law, stiction $\boldsymbol{\gamma}_{T}=\mathbf{0}$ occurs as long as the magnitude of the tangential force $\left\|\lambda_{T}\right\|$ is less than $\mu \lambda_{N}$, where $\mu$ is the friction coefficient. Furthermore, sliding $\boldsymbol{\gamma}_{T} \neq \mathbf{0}$ is possible if the magnitude is exactly $\mu \lambda_{N}$ and the tangential force acts in the opposite sliding direction. The mathematical formulation of the isotropic spatial Coulomb friction can be stated in the form of a normal cone inclusion

$$
-\boldsymbol{\gamma}_{T} \in \mathcal{N}_{\mu \lambda_{N} \mathcal{D}}\left(\boldsymbol{\lambda}_{T}\right)=\left\{\begin{array}{ll}
\{\boldsymbol{0}\} & \text { if }\left\|\boldsymbol{\lambda}_{T}\right\|<\mu \lambda_{N} \\
\mathbb{R}_{0}^{+} \boldsymbol{\lambda}_{T} & \text { if }\left\|\boldsymbol{\lambda}_{T}\right\|=\mu \lambda_{N}
\end{array},\right.
$$

where the convex set $\mu \lambda_{N} \mathcal{D}$ is the scaled two dimensional friction disc $\mathcal{D}=\left\{x \in \mathbb{R}^{2} \mid\|x\| \leq\right.$ 1 ) with radius $\mu \lambda_{N}$ as shown in Fig. 7(b). This formulation covers both the sticking and the slipping case. The sticking case corresponds to the situation where the tangential force $\lambda_{T}$ lies inside the scaled friction disc. In that case, the normal cone contains just the zero element. If, on the other hand, the force lies on the boundary of the disc, the normal cone becomes the outward normal ray of the disc. The fact that the friction force has to act in the opposite direction of the sliding velocity is reflected in the negative sign in front of the sliding velocity in the inclusion formulation (37).

\subsubsection{Impact laws}

Signorini's law (34) and Coulomb's friction law (37) are set-valued force laws for the nonimpulsive contact forces. In addition, as we consider hard unilateral constraints, impulsive contact forces arise whenever a contact closes with a negative relative velocity $\gamma_{N}^{-}<0$. Such a finite speed-of-approach requires a jump in the generalized velocities $\boldsymbol{u}$, which has to be induced by the impulsive contact forces $\boldsymbol{\Lambda}$ through the impact equation (21). The jump in generalized velocities has to be such that each post-impact velocity in normal direction is nonnegative, i.e., $\gamma_{N}^{+} \geq 0$ and, therefore, kinematically admissible.

The constitutive relation between the impulsive contact force and the kinematic quantities, which characterize the velocity jump is called an impact law. Various types of impact laws exist and the one adopted in this work is based on the classical Newtonian impact law $[19,35,37]$. In its extended form, it is also stated as a normal cone inclusion which for the normal direction has the form

$$
-\left(\gamma_{N}^{+}+\varepsilon_{N} \gamma_{N}^{-}\right) \in \mathcal{N}_{\mathbb{R}_{0}^{+}}\left(\Lambda_{N}\right)
$$

Here, $\gamma_{N}^{-}, \gamma_{N}^{+}$are the relative velocities in normal direction before and after the impact and $\varepsilon_{N} \in[0,1]$ is the coefficient of restitution for the normal direction. The case with $\varepsilon_{N}=1$ corresponds to a reflection of the normal velocity whereas a smaller $\varepsilon_{N}$ additionally dissipates energy. 
The inclusion (38) contains the two cases

$$
\begin{array}{ll}
\text { case 1: } & \gamma_{N}^{+}+\varepsilon_{N} \gamma_{N}^{-}=0 \wedge \Lambda_{N}>0, \\
\text { case 2: } & \gamma_{N}^{+}+\varepsilon_{N} \gamma_{N}^{-} \geq 0 \wedge \Lambda_{N}=0 .
\end{array}
$$

In the first case, there is an impact with impulsive contact force $\Lambda_{N}>0$ and the Newton impact law is fulfilled because $\gamma_{N}^{+}+\varepsilon_{N} \gamma_{N}^{-}=0$. For a kinematically incompatible preimpact velocity $\gamma_{N}^{-}<0$ the impact equation delivers a compatible post-impact velocity $\gamma_{N}^{+}=-\varepsilon_{N} \gamma_{N}^{-}>0$. In the second case, no impulsive contact force is exerted and the Newton impact law is only fulfilled as an inequality. This case accounts for the impact free separation of a contact, which may be induced by impacts at other contacts.

Normal contact forces induce tangential friction forces through Coulomb friction. Similarly, normal impulsive contact forces induce tangential impulsive friction forces. The tangential impact law has the form

$$
-\left(\boldsymbol{\gamma}_{T}^{+}+\varepsilon_{T} \boldsymbol{\gamma}_{T}^{-}\right) \in \mathcal{N}_{\mu \Lambda_{N} \mathcal{D}}\left(\boldsymbol{\Lambda}_{T}\right)
$$

The tangential parameter of restitution $\varepsilon_{T}$ can be used to model the dynamic effects of rubber superballs, which may store elastic energy in shear direction; see [19]. In the present work, we choose $\varepsilon_{T}=0$ for the rock terrain interaction as such elastic effects are clearly absent.

It is known that Newtonian impacts may lead to an increase in kinetic energy when combined with friction $[19,35]$. Such an energetic inconsistency may happen when the coefficients of restitution $\varepsilon_{N}$ and $\varepsilon_{T}$ of participating contacts are very different in value. This discrepancy of the Newtonian impact law is not really a burden for rockfall simulation as the normal restitution coefficient is typically very small and, therefore, close to $\varepsilon_{T}=0$. Furthermore, there exist pathological cases where a preimpact velocity $\gamma_{N}^{-}>0$ is mapped by the Newtonian impact law (38) onto a post-impact velocity $\gamma_{N}^{+}<0$ that lies no longer in the kinematically admissible set. Theoretically, such a preimpact state cannot be reached, however, it can occur in the numerics. Poisson's impact law, which is more elaborate as it uses two inequality complementarities, does not suffer from kinematic inconsistency [20].

\subsubsection{Combined contact model}

For a more compact notation, the inclusion formulations of the unilateral force law on velocity level (36) and the Coulomb friction law (37) are combined in a relation between the contact force $\lambda_{C}$ (31) and the relative contact velocity $\boldsymbol{\gamma}_{C}$ (24). The resulting combined force law can again be stated in the form of a (pseudo) normal cone inclusion

$$
-\boldsymbol{\gamma}_{C} \in \mathcal{N}_{\mathcal{S}}\left(\lambda_{C}\right)
$$

where the convex set $\mathcal{S}=\mathbb{R}_{0}^{+} \times \mu \lambda_{N} \mathcal{D}$ as a Cartesian product of the normal and tangential force reservoirs is introduced. Note that the set $\mathcal{S}$ is dependent on $\lambda_{N}$ and, therefore, on $\lambda_{C}$, which makes (41) a pseudo normal cone inclusion, being related to a pseudo-minimization problem [33]. Analogously, the impact laws are stated as

$$
-\left(\boldsymbol{\gamma}_{C}^{+}+\boldsymbol{\varepsilon} \boldsymbol{\gamma}_{C}^{-}\right) \in \mathcal{N}_{\mathcal{T}}\left(\boldsymbol{\Lambda}_{C}\right)
$$

where $\mathcal{T}=\mathbb{R}_{0}^{+} \times \mu \Lambda_{N} \mathcal{D}$ and $\boldsymbol{\varepsilon}=\operatorname{diag}\left(\varepsilon_{N}, \varepsilon_{T}, \varepsilon_{T}\right)$. 


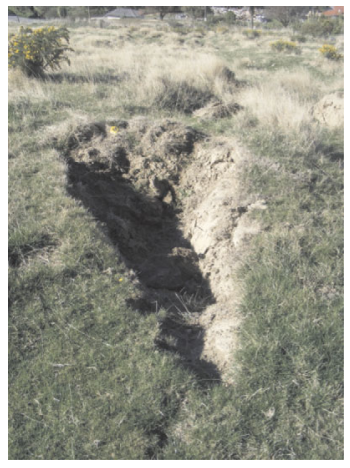

(a)

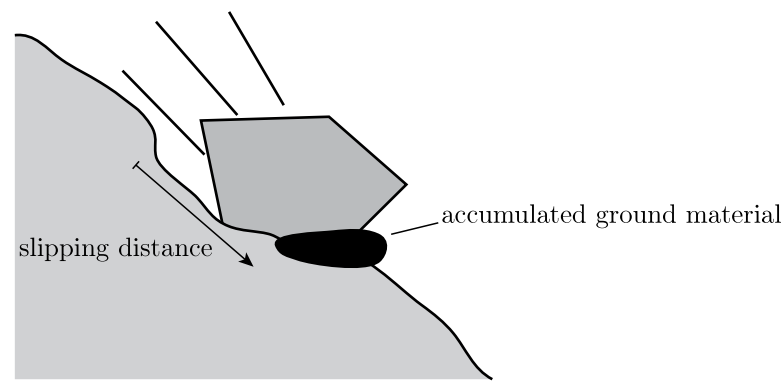

(b)

Fig. 8 The scarring phenomenon

For multiple contacts $C_{i}$, the inclusion problem of normal cone type can also be specified in a global form. Let $n_{c}$ be the number of active contacts on position level. The (pseudo) normal cone inclusion can be written as

$$
-\gamma \in \mathcal{N}_{\mathcal{C}}(\lambda)
$$

where

$$
\begin{gathered}
\boldsymbol{\gamma}=\left(\boldsymbol{\gamma}_{C_{1}}, \boldsymbol{\gamma}_{C_{2}}, \ldots, \boldsymbol{\gamma}_{C_{n_{c}}}\right)^{\mathrm{T}} \in \mathbb{R}^{3 n_{c}}, \quad \lambda=\left(\lambda_{C_{1}}, \lambda_{C_{2}}, \ldots, \lambda_{C_{n_{c}}}\right)^{\mathrm{T}} \in \mathbb{R}^{3 n_{c}} \\
\mathcal{C}=\mathcal{S}_{1} \times \mathcal{S}_{2} \times \cdots \times \mathcal{S}_{n_{c}} .
\end{gathered}
$$

A simplified global form can analogously be written for the impact laws.

\subsection{Slippage dependent friction model}

Field observations of rockfall events reveal the so-called "scarring" phenomenon. Upon impact with the terrain, the rock penetrates the terrain cover and ploughs a scar through the top-soil while sliding and the rock subsequently detaches from the terrain. A trace on the terrain with a finite scar length can then be observed. Figure 8(a) shows such a scar, where the scar starts at the tip of the triangular indentation (i.e., the bottom of the figure). While sliding, the rock continuously accumulates ground material in front of it (see Fig. 8(b)), which leads to an increase in sliding resistance. Such an increase in friction at the contact between rock and terrain causes the rock to topple and detach from the terrain. This phenomenon has a resemblance with an ice skater, which approaches a part of the ice surface covered with sand. The skate will suddenly experience much friction (possibly getting stuck) and the ice skater will be launched thereby losing contact with the ice. The modeling of the scarring behavior is therefore important for rockfall dynamics as it has influence on jump heights and distances. In order to describe the scarring phenomenon, the standard contact laws of Sect. 3.6 are complemented here with a novel model for the friction coefficient. A simulation showing the effect of the novel friction model and illustrating the scarring phenomenon is presented in Sect. 5.1.

The slippage dependent friction model extends the Coulomb friction model with a slippage dependent friction coefficient $\mu(s)$ where the slippage $s$ is an internal state of the 


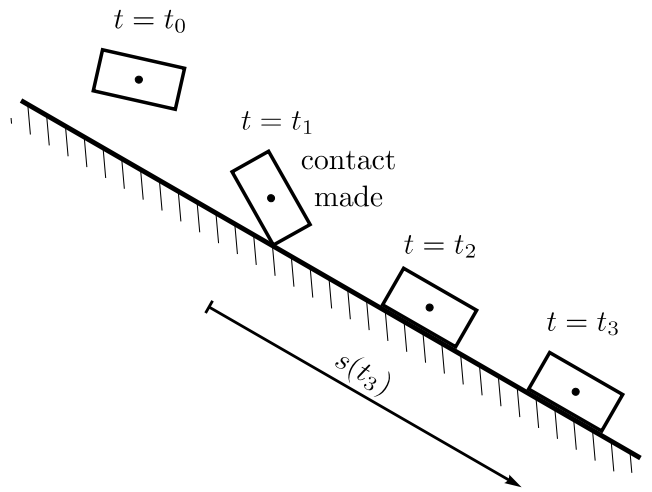

(a)

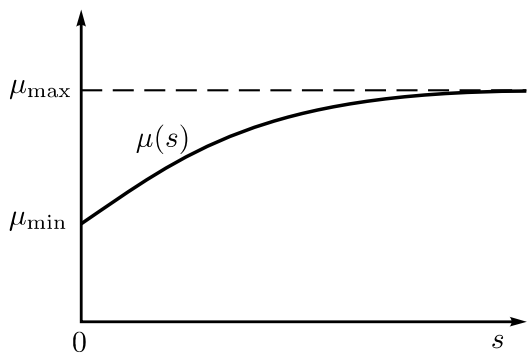

(b)

Fig. 9 Slippage dependent friction model

friction model. The slippage $s$ is loosely being defined as the distance which the center of mass has traveled during the contact phase (see Fig. 9(a)). The dependence of the friction coefficient on the slippage is chosen as

$$
\mu(s)=\mu_{\min }+\frac{2}{\pi}\left(\mu_{\max }-\mu_{\min }\right) \arctan (\kappa s),
$$

where $\mu_{\min }, \mu_{\max }$, and $\kappa$ are parameters of the friction model. The friction coefficient $\mu$ equals $\mu_{\min }$ for $s=0$ and tends to $\mu_{\max }$ for very large values of $s$, see Fig. 9(b). Hence, the frictional resistance increases with increasing slippage but saturates for very large values of the slippage.

The slippage distance $s$, being an additional state variable, has a time-evolution which is described by the differential equation

$$
\dot{s}= \begin{cases}\left\|v_{S}\right\| & \text { if at least one contact with terrain } \\ -\beta s & \text { else }\end{cases}
$$

and the additional initial condition $s(0)=s_{0} \geq 0$. As long as there is at least one active contact with $\lambda_{N}>0$ between terrain and rock, $s$ grows by integrating the norm of the center of mass velocity $\boldsymbol{v}_{S}$ of the rock. The slippage variable $s$ therefore increases with the slipping distance of the center of mass. An exponential decay to zero of the slippage $s$ is assumed when the rock is no longer in contact with the terrain. The speed of the decay is steered by the friction model parameter $\beta$. Note that the slippage $s$ is not simply set to zero upon contact loss between rock and terrain. The slippage variable $s$ changes continuously in the presented model, which reflects the physical behavior that the rock gradually has to overcome the heap of ground material in front of it. Furthermore, the continuous change of $s$ avoids numerical chattering problems.

\section{Numerical time-integration}

The main numerical integration techniques for systems with unilateral constraints are the event-driven integration method and the time-stepping method [1, 37, 41], of which the 
time-stepping method is far more robust than the former method. The event-driven integration method numerically integrates the equations of motion using a standard ODE or DAE solver up to a nonsmooth event (a collision or stick-slip transition) at which integration is halted. The impact equations are then applied to arrive at post-impact velocities and the new contact configuration (or "hybrid mode") is determined for which standard integration can be continued. The event-driven method has the disadvantage that it is not robust and that it can not overcome accumulations of nonsmooth events, i.e., when infinitely many events occur in a finite time. The time-stepping method, which has been introduced by Moreau [37], remedies these problems by choosing a blurred approach in which there is no real distinction between nonimpulsive and impulsive dynamics. Conventional integration methods determine accelerations from forces and use the accelerations to calculate velocity updates and position updates over time-steps. The basic idea behind the time-stepping method is to only calculate velocity updates and position updates over time-steps, usually with a fixed step-size. The algorithm calculates for each time-step the percussion, being the overall integral of contact forces and sums of impulsive contact forces over the time-step. Multiple events may take place during one time-step but only a single finite percussion is computed. The instantaneous value of the contact force or a single impulsive contact force is therefore never determined. Correspondingly, the acceleration is not computed by the algorithm, as it becomes infinite for impulsive forces. The positions and velocities at the end of the time-step are found by solving an algebraic inclusion, which describes the contact problem, for instance by formulating it as a linear or nonlinear complementarity problem or as a set of nonlinear equations by using the proximal point function (the so-called augmented Lagrangian approach) [2, 33]. The time-stepping method is especially useful when one is interested in the global motion of systems with many contact points, leading to a large number of events. Each individual event is for those applications not of importance but the global motion is determined by the sum of all events. The benefit of time-stepping methods over event-driven integration methods is the fact that no (or less) event-detection and index sets are needed. This makes the algorithm less complex, more robust, and will give a reduction in computation time when many contacts are involved. A second advantage of the time-stepping method is its capability to pass accumulation points of impacts. A notable disadvantage of the time-stepping method is its low-order accuracy.

The time-stepping scheme adopted in this work is based on the semi-implicit energetically consistent numerical scheme by Möller [36]. The contact problem in each time-step is formulated as a set of implicit equations by using the proximal point function and solved by a Gauss-Seidel iteration method. The discretized equations of motion and the contact inclusion problem are discussed in Sect. 4.1 and reformulated in their explicit form in Sect. 4.2. In Sect. 4.3, the discrete inclusion problem is formulated as an implicit relation for the contact percussions and are transformed into nonlinear equations.

\subsection{Discretization of the equations of motion}

The semi-implicit energetically consistent time-stepping scheme by Möller (Sect. 5.2.5 in [36]) is an adaptation of the classical Moreau time-stepping scheme [37] in which the gyroscopic terms are discretized in a special way such that the scheme becomes energetically consistent. Here, a slightly more explicit version of Möller's scheme is chosen which carries many of the energetic consistency properties and is more computational efficient. The merit of an energetically consistent scheme for rockfall dynamics is that the rotational energy of the rock remains constant during flight phases.

The integration scheme employs a midpoint method (see Fig. 10) with time-step size $\Delta t$ such that the generalized positions are approximated at the beginpoint $t^{\mathrm{B}}$ and endpoint 
Fig. 10 Illustration of the midpoint scheme

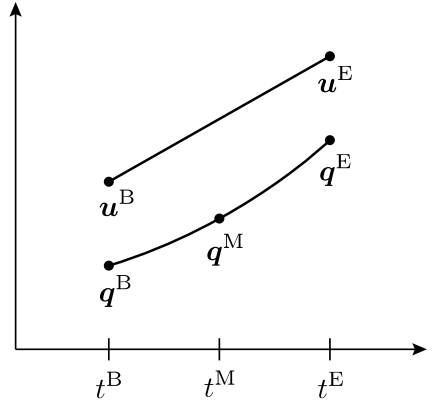

$t^{\mathrm{E}}=t^{\mathrm{B}}+\Delta t$ of the time-step as well as at a midpoint $t^{\mathrm{M}}=t^{\mathrm{B}}+\frac{\Delta t}{2}$. The time-stepping scheme calculates for a given $\left(\boldsymbol{q}^{\mathrm{B}}, \boldsymbol{u}^{\mathrm{B}}\right)$ at the beginning of the time-step $t_{B}$ an approximant for the state $\left(\boldsymbol{q}^{\mathrm{E}}, \boldsymbol{u}^{\mathrm{E}}\right)$ as well as an approximant $\boldsymbol{P} \approx \int_{\left[t^{\mathrm{B}}, t^{\mathrm{E}}\right]} \mathrm{d} \boldsymbol{P}$ of the percussion during the time-step.

Given the state of the system $\left(\boldsymbol{q}^{\mathrm{B}}, \boldsymbol{u}^{\mathrm{B}}\right)$ at the beginning of the time-step $t^{\mathrm{B}}$ a half-explicit Euler step in the generalized coordinates $\boldsymbol{q}$ is performed to arrive at the midpoint $t^{\mathrm{M}}$. The generalized coordinates $\boldsymbol{q}^{\mathrm{M}}$ at the midpoint are used to evaluate the generalized force directions $\boldsymbol{W}\left(\boldsymbol{q}^{\mathrm{M}}\right)$. These are necessary to set up the inclusion problem, which is subsequently solved for the contact percussions $\boldsymbol{P}$ acting during this time-step. Using the contact percussions $\boldsymbol{P}$, the velocity $\boldsymbol{u}^{\mathrm{E}}$ at the end $t^{\mathrm{E}}$ of the time-step is determined and a second explicit Euler step is performed to obtain the end configuration $\boldsymbol{q}^{\mathrm{E}}$. The differential measure equation (22) and the relations between the generalized velocity and the time differential of the generalized coordinates (14) are discretized in the following way:

$$
\begin{aligned}
& \boldsymbol{q}^{\mathrm{M}}=\boldsymbol{q}^{\mathrm{B}}+\frac{\Delta t}{2} \boldsymbol{F}\left(\boldsymbol{p}_{I K}^{\mathrm{B}}\right) \boldsymbol{u}^{\mathrm{B}}, \\
& \boldsymbol{M}\left(\boldsymbol{u}^{\mathrm{E}}-\boldsymbol{u}^{\mathrm{B}}\right)+\Delta t\left(\begin{array}{c}
c_{d}\left({ }_{I} \boldsymbol{r}_{O S}^{\mathrm{B}}\right)_{I} \boldsymbol{v}_{S}^{\mathrm{B}}+m g_{I} \boldsymbol{e}_{z}^{I} \\
\left.{ }_{\frac{1}{2}\left({ }_{K}\right.} \widetilde{\boldsymbol{\Omega}}^{\mathrm{B}}{ }_{K} \boldsymbol{\Theta}_{S}+{ }_{K} \boldsymbol{\Theta}_{S K} \widetilde{\boldsymbol{\Omega}}^{\mathrm{B}}\right)\left({ }_{K} \boldsymbol{\Omega}^{\mathrm{E}}+{ }_{K} \boldsymbol{\Omega}^{\mathrm{B}}\right)
\end{array}\right)-\boldsymbol{W}\left(\boldsymbol{q}^{\mathrm{M}}\right) \boldsymbol{P}=0, \\
& \boldsymbol{q}^{\mathrm{E}}=\boldsymbol{q}^{\mathrm{B}}+\Delta t \boldsymbol{F}\left(\boldsymbol{p}_{I K}^{\mathrm{M}}\right) \frac{\boldsymbol{u}^{\mathrm{E}}+\boldsymbol{u}^{\mathrm{B}}}{2},
\end{aligned}
$$

where the drag force term in (49) is discretized explicitly and the gyroscopic term semiimplicitly (see [36]). The rationale behind this is discussed at the end of this section.

The differential equation for the slipping distance (47) is handled separately and also discretized explicitly as

$$
s^{\mathrm{E}}= \begin{cases}s^{\mathrm{B}}+\Delta t\left\|\boldsymbol{v}_{S}^{\mathrm{E}}\right\| & \text { if contact, } \\ s^{\mathrm{B}} e^{-\beta \Delta t} & \text { otherwise. }\end{cases}
$$

The time-stepping equations (49) are solved together with the discretized contact force/ impact laws for the percussions $\boldsymbol{P}$ which can be expressed by the inclusion problem

$$
-\left(\boldsymbol{\gamma}^{\mathrm{E}}+\boldsymbol{\varepsilon} \boldsymbol{\gamma}^{\mathrm{B}}\right) \in \mathcal{N}_{\mathcal{C}}(\boldsymbol{P}),
$$

with the kinematic relations

$$
\boldsymbol{\gamma}^{\mathrm{E}}=\boldsymbol{W}\left(\boldsymbol{q}^{\mathrm{M}}\right)^{\mathrm{T}} \boldsymbol{u}^{\mathrm{E}}, \quad \boldsymbol{\gamma}^{\mathrm{B}}=\boldsymbol{W}\left(\boldsymbol{q}^{\mathrm{M}}\right)^{\mathrm{T}} \boldsymbol{u}^{\mathrm{B}} .
$$


The energetic consistency properties of the scheme can be checked by pre-multiplying (49) with $\frac{1}{2}\left(\boldsymbol{u}^{\mathrm{E}}+\boldsymbol{u}^{\mathrm{B}}\right)^{\mathrm{T}}$, which gives the energy balance

$$
\Delta T+\Delta V-\Delta E_{\mathrm{gyro}}-\Delta E_{\mathrm{drag}}-\Delta E_{\text {constr }}=0,
$$

where $\Delta T=\frac{1}{2}\left(\boldsymbol{u}^{\mathrm{E}}+\boldsymbol{u}^{\mathrm{B}}\right)^{\mathrm{T}} \boldsymbol{M}\left(\boldsymbol{u}^{\mathrm{E}}-\boldsymbol{u}^{\mathrm{B}}\right)=T^{\mathrm{E}}-T^{\mathrm{B}}$ is the change in kinetic energy and $\Delta V=$ $-\frac{1}{2} m g\left({ }_{I} \boldsymbol{v}_{S}^{\mathrm{E}}+{ }_{I} \boldsymbol{v}_{S}^{\mathrm{B}}\right)^{\mathrm{T}}{ }_{I} \boldsymbol{e}_{z}^{I} \Delta t=-m g\left(\boldsymbol{q}^{\mathrm{E}}-\boldsymbol{q}^{\mathrm{B}}\right)^{\mathrm{T}}{ }_{I} \boldsymbol{e}_{z}^{I}=V^{\mathrm{E}}-V^{\mathrm{B}}$ is the change in gravitational potential energy. Of interest is the energy increment of the gyroscopic forces

$$
\Delta E_{\text {gyro }}=-\frac{1}{4}\left({ }_{K} \boldsymbol{\Omega}^{\mathrm{E}}+{ }_{K} \boldsymbol{\Omega}^{\mathrm{B}}\right)^{\mathrm{T}}\left({ }_{K} \widetilde{\boldsymbol{\Omega}}^{\mathrm{B}}{ }_{K} \boldsymbol{\Theta}_{S}+{ }_{K} \boldsymbol{\Theta}_{S K} \widetilde{\boldsymbol{\Omega}}^{\mathrm{B}}\right)\left({ }_{K} \boldsymbol{\Omega}^{\mathrm{E}}+{ }_{K} \boldsymbol{\Omega}^{\mathrm{B}}\right) \Delta t=0
$$

which vanishes because of the skew-symmetry of the matrix $\left({ }_{K} \widetilde{\boldsymbol{\Omega}}^{\mathrm{B}}{ }_{K} \boldsymbol{\Theta}_{S}+{ }_{K} \boldsymbol{\Theta}{ }_{S K} \widetilde{\boldsymbol{\Omega}}^{\mathrm{B}}\right)$. This favorable property of the scheme is due to the nonstandard discretization of the gyroscopic term in (49), an idea which has also been used in [36], Sect. 5.2.5. However, the energy increment due to drag

$$
\Delta E_{\mathrm{drag}}=-\frac{1}{2} c_{d}\left({ }_{I} \boldsymbol{r}_{O S}^{\mathrm{B}}\right)\left({ }_{I} \boldsymbol{v}_{S}^{\mathrm{E}}+{ }_{I} \boldsymbol{v}_{S}^{\mathrm{B}}\right)^{\mathrm{T}}{ }_{I} \boldsymbol{v}_{S}^{\mathrm{B}} \Delta t
$$

is not guaranteed to be negative due to the fully explicit discretization. A more implicit discretization of this uncritical term can of course remedy this small impediment at the cost of computational efficiency. Finally, the energy increment of the contact percussions

$$
\Delta E_{\text {constr }}=\frac{1}{2}\left(\boldsymbol{u}^{\mathrm{E}}+\boldsymbol{u}^{\mathrm{B}}\right)^{\mathrm{T}} \boldsymbol{W} \boldsymbol{P}=\frac{1}{2}\left(\boldsymbol{\gamma}^{\mathrm{E}}+\boldsymbol{\gamma}^{\mathrm{B}}\right)^{\mathrm{T}} \boldsymbol{P}
$$

is exactly the contact work of the contact percussions. Hence, $\Delta E_{\text {constr }}$ is negative if the contact force/impact law (52) is dissipative.

\subsection{Explicit form of the time-stepping equations}

In each time-step, the discretized equations of motion (49) have to be solved for $\boldsymbol{u}^{\mathrm{E}}$. The translational and rotational equations of motion (49) are therefore written as linear functions in $\boldsymbol{u}^{\mathrm{E}}$. Hereto, the generalized force direction $\boldsymbol{W}$ is split into its translational and rotational contributions

$$
\boldsymbol{W}=\left(\begin{array}{c}
W_{v} \\
W_{\Omega}
\end{array}\right)
$$

where $\boldsymbol{W}_{v}$ corresponds to the upper three rows of the generalized force directions $\boldsymbol{W}$ that contribute to the translational part of the equations of motion and $\boldsymbol{W}_{\boldsymbol{\Omega}}$ corresponds to the three rows for the rotational part. In the following, the coordinate frames in which the mathematical objects are represented have been omitted which simplifies the notation.

For the translational degrees of freedom, solving (49) for $\boldsymbol{v}^{\mathrm{E}}$ leads to

$$
\boldsymbol{v}^{\mathrm{E}}=\boldsymbol{v}^{\mathrm{B}} \underbrace{-\Delta t\left(m^{-1} c_{d} \boldsymbol{v}^{\mathrm{B}}+g \boldsymbol{e}_{z}\right)}_{\Delta \boldsymbol{v}_{\text {free }}}+m^{-1} \boldsymbol{W}_{\boldsymbol{v}} \boldsymbol{P}=\boldsymbol{v}^{\mathrm{B}}+\Delta \boldsymbol{v}_{\text {free }}+m^{-1} \boldsymbol{W}_{\boldsymbol{v}} \boldsymbol{P} .
$$

Herein, the abbreviation $\Delta \boldsymbol{v}_{\text {free }}$ has been introduced for the free flight contributions, consisting only of the velocity change due to gravity and linear dissipation. 
Solving the discretized equations of motion for the rotational degrees of freedom is more involved. After the separation of the terms for $\boldsymbol{\Omega}^{\mathrm{B}}$ and $\boldsymbol{\Omega}^{\mathrm{E}}$, the rotational equation of motion in (49) becomes

$$
\left(\boldsymbol{\Theta}+\frac{\Delta t}{2} \widetilde{\boldsymbol{\Theta}}\right) \boldsymbol{\Omega}^{\mathrm{E}}=\left(\boldsymbol{\Theta}-\frac{\Delta t}{2} \widetilde{\boldsymbol{\Theta}}\right) \boldsymbol{\Omega}^{\mathrm{B}}+\boldsymbol{W}_{\boldsymbol{\Omega}} \boldsymbol{P},
$$

where the skew-symmetric abbreviation $\widetilde{\boldsymbol{\Theta}}=\widetilde{\boldsymbol{\Omega}}^{\mathrm{B}} \boldsymbol{\Theta}+\boldsymbol{\Theta} \widetilde{\boldsymbol{\Omega}}^{\mathrm{B}}$ is introduced and in which the subscript $K$ has been dropped for brevity. In order to arrive at the same structure as for the translational stepping equations (59), the zero term $\Delta t \widetilde{\boldsymbol{\Theta}} \boldsymbol{\Omega}^{\mathrm{B}}-\Delta t \widetilde{\boldsymbol{\Theta}} \boldsymbol{\Omega}^{\mathrm{B}}=0$ is added on the right-hand side of (60) leading to

$$
\left(\boldsymbol{\Theta}+\frac{\Delta t}{2} \widetilde{\boldsymbol{\Theta}}\right) \boldsymbol{\Omega}^{\mathrm{E}}=\left(\boldsymbol{\Theta}+\frac{\Delta t}{2} \widetilde{\boldsymbol{\Theta}}\right) \boldsymbol{\Omega}^{\mathrm{B}}-\Delta t \widetilde{\boldsymbol{\Theta}} \boldsymbol{\Omega}^{\mathrm{B}}+\boldsymbol{W}_{\boldsymbol{\Omega}} \boldsymbol{P} .
$$

After introducing the additional abbreviation

$$
\boldsymbol{\Theta}_{\Delta}:=\boldsymbol{\Theta}+\frac{\Delta t}{2} \widetilde{\boldsymbol{\Theta}},
$$

which can be interpreted as a modified numerical inertia matrix, and solving for $\boldsymbol{\Omega}^{\mathrm{E}}$ by pre-multiplying with $\boldsymbol{\Theta}_{\Delta}^{-1}$, the angular velocity difference equation takes the form

$$
\boldsymbol{\Omega}^{\mathrm{E}}=\boldsymbol{\Omega}^{\mathrm{B}} \underbrace{-\Delta t \boldsymbol{\Theta}_{\Delta}^{-1} \widetilde{\boldsymbol{\Theta}} \boldsymbol{\Omega}^{\mathrm{B}}}_{\Delta \boldsymbol{\Omega}_{\text {free }}}+\boldsymbol{\Theta}_{\Delta}^{-1} \boldsymbol{W}_{\boldsymbol{\Omega}} \boldsymbol{P}=\boldsymbol{\Omega}^{\mathrm{B}}+\Delta \boldsymbol{\Omega}_{\text {free }}+\boldsymbol{\Theta}_{\Delta}^{-1} \boldsymbol{W}_{\boldsymbol{\Omega}} \boldsymbol{P} .
$$

Herein, the term $\Delta \boldsymbol{\Omega}_{\text {free }}$ contains the free flight contributions which are due to gyroscopic effects.

The stepping equations (59) and (63) for the linear and angular velocity are assembled in stepping equations for the generalized velocity $\boldsymbol{u}$. Using the assembled mass matrix $\boldsymbol{M}_{\Delta}$ and the free flight term $\Delta \boldsymbol{u}_{\text {free }}$

$$
\boldsymbol{M}_{\Delta}=\left(\begin{array}{cc}
m \boldsymbol{I}_{3 \times 3} & \boldsymbol{O}_{3 \times 3} \\
\boldsymbol{O}_{3 \times 3} & \boldsymbol{\Theta}_{\Delta}
\end{array}\right), \quad \Delta \boldsymbol{u}_{\text {free }}=\left(\begin{array}{c}
\Delta \boldsymbol{v}_{\text {free }} \\
\Delta \boldsymbol{\Omega}_{\text {free }}
\end{array}\right)
$$

the stepping equations for the generalized velocity $\boldsymbol{u}$ read as

$$
\boldsymbol{u}^{\mathrm{E}}=\boldsymbol{u}^{\mathrm{B}}+\Delta \boldsymbol{u}_{\text {free }}+\boldsymbol{M}_{\Delta}^{-1} \boldsymbol{W} \boldsymbol{P} .
$$

\subsection{Discrete inclusion problem}

In each time-step, the stepping equations (65) are solved together with the inclusion (52)

$$
-\left(\boldsymbol{\gamma}^{\mathrm{E}}+\boldsymbol{\varepsilon} \boldsymbol{\gamma}^{\mathrm{B}}\right) \in \mathcal{N}_{\mathcal{C}}(\boldsymbol{P})
$$

which contains the combined contact and impact laws in discretized form. Hereto, the contact velocity $\boldsymbol{\gamma}^{\mathrm{E}}=\boldsymbol{W}^{\mathrm{T}} \boldsymbol{u}^{\mathrm{E}}$ is expressed in the percussion $\boldsymbol{P}$ using the stepping equations (65), which leads to

$$
\begin{aligned}
\boldsymbol{\gamma}^{\mathrm{E}} & =\boldsymbol{W}^{\mathrm{T}}\left(\boldsymbol{u}^{\mathrm{B}}+\Delta \boldsymbol{u}_{\text {free }}+\boldsymbol{M}_{\Delta}^{-1} \boldsymbol{W} \boldsymbol{P}\right) \\
& =\boldsymbol{W}^{\mathrm{T}} \boldsymbol{M}_{\Delta}^{-1} \boldsymbol{W} \boldsymbol{P}+\boldsymbol{W}^{\mathrm{T}}\left(\Delta \boldsymbol{u}_{\text {free }}+\boldsymbol{u}^{\mathrm{B}}\right) .
\end{aligned}
$$




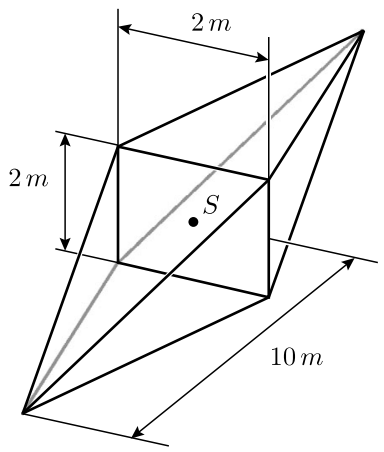

(a)

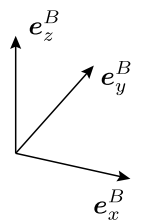

(b)

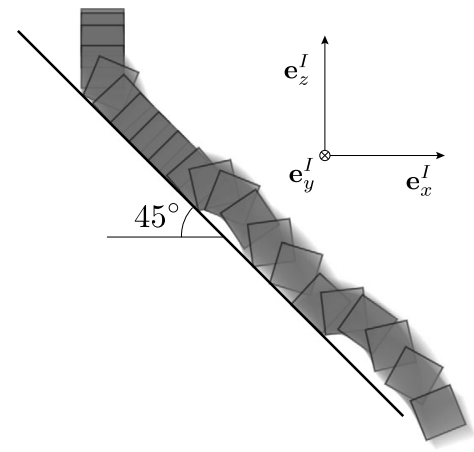

Fig. 11 (a) The block geometry: A nonregular octahedron, e.g., two pyramids with a regular quadrilateral base. (b) Stroboscopic image of a simulation with slipping distance dependent $\mu$

The expression $\boldsymbol{\gamma}^{\mathrm{E}}+\boldsymbol{\varepsilon} \boldsymbol{\gamma}^{\mathrm{B}}$ in the inclusion problem (52) can therefore be written as

$$
\boldsymbol{\gamma}^{\mathrm{E}}+\boldsymbol{\varepsilon} \boldsymbol{\gamma}^{\mathrm{B}}=\underbrace{\boldsymbol{W}^{\mathrm{T}} \boldsymbol{M}_{\Delta}^{-1} \boldsymbol{W}}_{\boldsymbol{G}} \boldsymbol{P}+\underbrace{\boldsymbol{W}^{\mathrm{T}}\left(\Delta \boldsymbol{u}_{\mathrm{free}}+(1+\boldsymbol{\varepsilon}) \boldsymbol{u}^{\mathrm{B}}\right)}_{\boldsymbol{c}},
$$

in which the Delassus matrix $\boldsymbol{G}$ and the vector $\boldsymbol{c}$ have been introduced. The inclusion problem (52) can therefore be written as

$$
-(\boldsymbol{G P}+\boldsymbol{c}) \in \mathcal{N}_{\mathcal{C}}(\boldsymbol{P}),
$$

in which $\boldsymbol{P}$ is the only unknown. Using theory from convex analysis [35], we can transform the inclusion problem to an implicit proximal point equation

$$
\boldsymbol{P}=\operatorname{prox}_{\mathcal{C}}^{R}\left(\boldsymbol{P}-\boldsymbol{R}^{-1}(\boldsymbol{G P}+\boldsymbol{c})\right) .
$$

Herein, $\operatorname{prox}_{\mathcal{C}}^{R}(\cdot)$ is the proximal point operator on the convex set $\mathcal{C}$ in the metric $\boldsymbol{R}=\boldsymbol{R}^{\mathrm{T}}>$ 0 . The proximal point equation (69) is implicit in the contact percussions $\boldsymbol{P}$. Iterative methods can be used to solve for the contact percussions $\boldsymbol{P}[1,40]$. Finally, the generalized velocity $\boldsymbol{u}^{\mathrm{E}}$ can be computed with the stepping equation (65).

\section{Simulations}

This section documents two simulations, which show the capabilities of simulation techniques presented in this paper and clearly demonstrate the importance of the influence of rock shape on rockfall trajectory simulation. First, a simulation which illustrates the effect of the slippage dependent friction model is presented in Sect. 5.1. A simulation which shows the stabilization around the principal axis of largest inertia is discussed in Sect. 5.2.

\subsection{Slippage dependent friction model: Simulation}

In Sect. 3.7, the so-called "scarring" phenomenon has been explained: Upon impact with the terrain, the rock does not immediately rebounce, but slides over the terrain, accumulating 
ground material in front of it which increases its resistance, causing it to topple and detach from the terrain. To illustrate the scarring phenomenon and the behavior of the slippage dependent friction model, introduced in Sect. 3.7, the motion of a planar square block is considered which descends along an inclined slope of $45^{\circ}$ (see Fig. 11(b)). For demonstration and visualization purposes, the example is chosen to be essentially a planar problem. However, the RAMMS:rockfall module which uses the theory and methods of the current paper is a fully three-dimensional code. The geometry of the body is chosen such that the relevant dynamics can be represented in $2 \mathrm{D}$ although the simulation is actually performed in $3 \mathrm{D}$. Hereto, the motion on an inclined plane is considered of a nonregular octahedron, being two pyramids with a square base (see Fig. 11(a)). Only the square base of the octahedron comes into contact with the inclined plane. The large width of the octahedron increases the inertia around the in-plane axes and therefore stabilizes the planar motion.

The geometry of the body is specified in the body-fixed frame $B$. The octahedron has a square base with dimensions $2 \mathrm{~m} \times 2 \mathrm{~m}$, which lies in the $\boldsymbol{e}_{x}^{B}-\boldsymbol{e}_{z}^{B}$-plane and a width of $10 \mathrm{~m}$ in the $\boldsymbol{e}_{y}^{B}$-direction. Assuming a homogeneous density $\rho=3000 \mathrm{~kg} \mathrm{~m}^{-3}$, the mass becomes $m=80000 \mathrm{~kg}$ and the moments of inertia are $\Theta_{y}=32,000 \mathrm{~kg} \mathrm{~m}^{2}$ and $\Theta_{x}=\Theta_{z}=$ $816,000 \mathrm{~kg} \mathrm{~m}^{2}$ corresponding to the principal axis of inertia $\boldsymbol{e}_{x}^{B}, \boldsymbol{e}_{y}^{B}$ and $\boldsymbol{e}_{z}^{B}$, respectively. The terrain consists of a plane with slope angle $\alpha=45^{\circ}$. As the scarring phenomenon does not involve an immediate rebounce, the coefficients of restitution for the Newtonian impact law are chosen completely inelastic (e.g., $\varepsilon_{N}=\varepsilon_{T}=0$ ). The slippage dependent friction coefficient $\mu(s)$ runs from $\mu_{\min }=0.2$ to $\mu_{\max }=1.3$. The slippage decay parameter $\beta=$ $20 \mathrm{~s}^{-1}$ and sliding distance friction proportionality constant $\kappa=0.7 \mathrm{~m}^{-1}$ are used. The gravitational acceleration is chosen as $g=9.81 \mathrm{~m} \mathrm{~s}^{-2}$.

A projection of the resulting motion onto the inertial $\boldsymbol{e}_{x}^{I}-\boldsymbol{e}_{z}^{I}$-plane is shown in Fig. 11(b). The body is released slightly above the slope; its body-fixed frame aligned with the inertial frame. Upon impact with the slope, it quickly settles into a slip motion, because of the completely inelastic impact law $\left(\varepsilon_{N}=0\right)$. Hence, a slip phase is commenced during which two corner points of the square base of the body are in contact with the slope. The body is sliding along the slope during this slip phase and the static force equilibrium in normal direction to the plane

$$
\lambda_{N 1}+\lambda_{N 2}=m g \cos \alpha
$$

holds, where $\lambda_{N 1}$ and $\lambda_{N 2}$ are the normal contact forces of the left and right contact point, respectively. Furthermore, the body is not rotating during the slip phase and the static torque equilibrium around the center of mass

$$
\left(\lambda_{N 1}-\lambda_{N 2}-\lambda_{T 1}-\lambda_{T 2}\right) r=0
$$

has to hold, where $r=1 \mathrm{~m}$ is half the width of the square base. The tangential contact forces $\lambda_{T 1}$ and $\lambda_{T 2}$, which obey Coulomb's friction law $\lambda_{T i} \in \mu \lambda_{N i} \operatorname{Sign} \gamma_{T i}$, amount to $\lambda_{T 1}=-\mu \lambda_{N 1}$ and $\lambda_{T 2}=-\mu \lambda_{N 2}$ as $\gamma_{T i}<0$ for the downward sliding along the slope. Solving these equations for the normal contact forces yields

$$
\lambda_{N 1}=\frac{1}{2}(1-\mu) m g \cos \alpha, \quad \lambda_{N 2}=\frac{1}{2}(1+\mu) m g \cos \alpha .
$$

Considering that $\lambda_{N 1} \geq 0$ has to hold for the left contact to remain closed, leads to the condition $\mu \leq 1$. As the body keeps sliding down the slope, the slippage $s$ increases and the value of $\mu(s)$ increases in accordance with the slippage dependent friction model (46):

$$
\mu(s)=\mu_{\min }+\frac{2}{\pi}\left(\mu_{\max }-\mu_{\min }\right) \arctan (\kappa s) .
$$


Eventually, the condition $\mu \leq 1$ is violated. Because $\lambda_{N 1}$ cannot become negative, the static torque equilibrium is no longer valid and the left corner point loses contact, i.e., contact point 1 opens. Thus, the block starts rotating. A second slip phase commences during which there is only contact at the right corner point of the square base. The equations of motion in this mode are

$$
\begin{aligned}
m \ddot{x} & =\lambda_{N 2} \sin \alpha+\lambda_{T 2} \cos \alpha=(\sin \alpha-\mu \cos \alpha) \lambda_{N 2}, \\
m \ddot{z} & =\lambda_{N 2} \cos \alpha-\lambda_{T 2} \sin \alpha-m g=(\cos \alpha+\mu \sin \alpha) \lambda_{N 2}-m g, \\
\Theta_{y} \ddot{\varphi} & =\lambda_{N 2} r(\sin \varphi-\cos \varphi)-\lambda_{T 2} r(\sin \varphi+\cos \varphi)=\lambda_{N 2} r((1+\mu) \sin \varphi-(1-\mu) \cos \varphi) .
\end{aligned}
$$

The normal contact velocity of the right contact point

$$
\gamma_{N 2}=\sin \alpha \dot{x}+\cos \alpha \dot{z}+r(\sin \varphi-\cos \varphi) \dot{\varphi}
$$

vanishes during the second slip phase. Differentiation gives the normal contact acceleration

$$
\dot{\gamma}_{N 2}=\sin \alpha \ddot{x}+\cos \alpha \ddot{z}+r(\sin \varphi-\cos \varphi) \ddot{\varphi}+r(\cos \varphi+\sin \varphi) \dot{\varphi}^{2} .
$$

Substitution of the equations of motion (73) into (75) yields the expression

$$
\dot{\gamma}_{N 2}=\left(\frac{1}{m}+\frac{r^{2}}{\Theta_{y}}(1-\sin 2 \varphi-\mu \cos 2 \varphi)\right) \lambda_{N 2}-g \cos \alpha+r(\cos \varphi+\sin \varphi) \dot{\varphi}^{2},
$$

which is linear in the contact force $\lambda_{N 2}$. The contact acceleration $\dot{\gamma}_{N 2}$ and contact force $\lambda_{N 2}$ are, for a closed contact with $\gamma_{N 2}=0$, inequality complementarity variables satisfying the Signorini condition

$$
0 \leq \dot{\gamma}_{N 2} \perp \lambda_{N 2} \geq 0
$$

on acceleration level. The linear equation (76) together with the inequality complementarity (77) constitutes the linear complementarity problem

$$
\begin{aligned}
& \dot{\gamma}_{N 2}=A(\varphi, \mu) \lambda_{N 2}+b(\varphi, \dot{\varphi}) \\
& 0 \leq \dot{\gamma}_{N 2} \perp \lambda_{N 2} \geq 0
\end{aligned}
$$

with

$$
\begin{aligned}
& A(\varphi, \mu)=\frac{1}{m}\left(1+\frac{1}{k^{2}}(1-\sin 2 \varphi-\mu \cos 2 \varphi)\right), \\
& b(\varphi, \dot{\varphi})=-g \cos \alpha+r(\cos \varphi+\sin \varphi) \dot{\varphi}^{2}
\end{aligned}
$$

and the abbreviation

$$
k^{2}=\frac{\Theta_{y}}{m r^{2}} .
$$

One may easily verify that $A(\varphi, \mu)>0$ for all $\varphi$ if $\mu<\mu_{P}$, where

$$
\mu_{P}=\sqrt{k^{4}+2 k^{2}}
$$

is a critical friction coefficient. If $\mu>\mu_{P}$, then there exists an interval of $\varphi$ for which $A(\varphi, \mu)<0$. The linear complementarity problem has for $A(\varphi, \mu)<0$ and $b(\varphi, \dot{\varphi})>0$ 
multiple solutions and for $A(\varphi, \mu)<0$ and $b(\varphi, \dot{\varphi})<0$ no solution. The phenomenon, which occurs for values of $\mu$ larger than the critical friction coefficient $\mu_{P}$, is known as the Painlevé paradox [32]. The contact force during the second slip phase is given by ratio

$$
\lambda_{N 2}=-\frac{b(\varphi, \dot{\varphi})}{A(\varphi, \mu)},
$$

with $A(\varphi, \mu)>0$ and $b(\varphi, \dot{\varphi})<0$. The angle $\varphi(t)$ increases during the slip phase and the value of $A(\varphi, \mu)$ lowers whereas $b(\varphi, \dot{\varphi})$ rises, i.e. both tend toward zero. Typically, the contact force first drastically increases. Contact may be lost through two different scenarios

1. The value of $b(\varphi, \dot{\varphi})$ vanishes whereas $A(\varphi, \mu)>0$. This means that the increase of the contact force is followed by a rapid decrease to zero. The block simply detaches from the slope as the contact force vanishes.

2. The value of $A(\varphi, \mu)$ vanishes, whereas $b(\varphi, \dot{\varphi}) \neq 0$. The contact force tends to infinity but with a bounded impulse. This is known as a frictional catastrophe. The contact opens impulsively, although no collision occurred.

In [15], it has been proven that the second scenario may only occur if the friction coefficient is sufficiently larger than the critical value $\mu_{P}$ of the Painlevé paradox. Performing a similar calculation as in [15], we obtain the critical value for the frictional catastrophe

$$
\mu_{C}=2 \sqrt{\frac{k^{4}+2 k^{2}}{3}}=\frac{2}{\sqrt{3}} \mu_{P} .
$$

For the chosen parameter values, it holds that $k^{2}=\frac{2}{3}$ which yields the critical friction coefficients $\mu_{P} \approx 0.9798$ and $\mu_{C} \approx 1.1314$. The maximal friction coefficient $\mu_{\max }=1.3$ is larger than $\mu_{C}$ and a frictional catastrophe may for certain initial conditions occur. However, in the presented simulation, the second slip phase ends with a simple detachment. The block enters a free flight phase during which the slipping distance $s$ decays exponentially with the exponential time constant $\tau=\beta^{-1}$. Upon the next impact, the same process starts all over again.

\subsection{Stabilization around the principal axis of largest inertia}

As observed in the field, rocks jumping down a hill build up rotational speed and tend to stabilize their angular velocity around the principal axis of largest inertia. Loosely speaking, tabular shaped rocks tend to roll down like a wheel, especially on meadow-type terrain. This phenomenon is also reported in [16] and it is suggested that tabular shaped rocks gradually become rounded and wheel-like due to sharp corners breaking off during the descent.

In order to give a physical explanation for the observed phenomenon, one may try to compare it with a similar idealized mechanical problem. It is readily known that the up-right rolling of a disk on a rough plane is stable above a critical speed, i.e., for a homogeneous thin disk with radius $r$ the critical rotation speed is given by $\Omega_{c}^{2}=\frac{1}{3} \frac{g}{r}$ [4]. However, this does not fully explain that also a tabular shaped rock, which does not roll but rather bumps down the slope, also stabilizes around the principal axis of largest inertia. In this respect, it is noteworthy to consider a freely rotating body, e.g. a rock in the air, which is known to have stable body rotations around the smallest and largest principal axes of inertia whereas the rotation around the middle axis is unstable. Moreover, the stability of the rolling motion of an ellipsoidal body on a rough plane has been shown to be governed by parametric resonance phenomena as the periodic rise and fall of the center of mass induces a parametric 


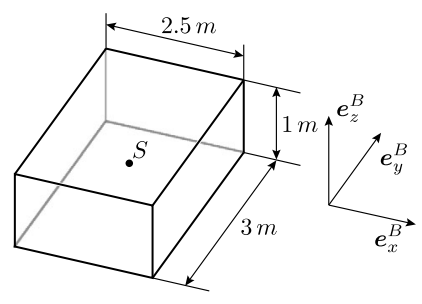

(a)

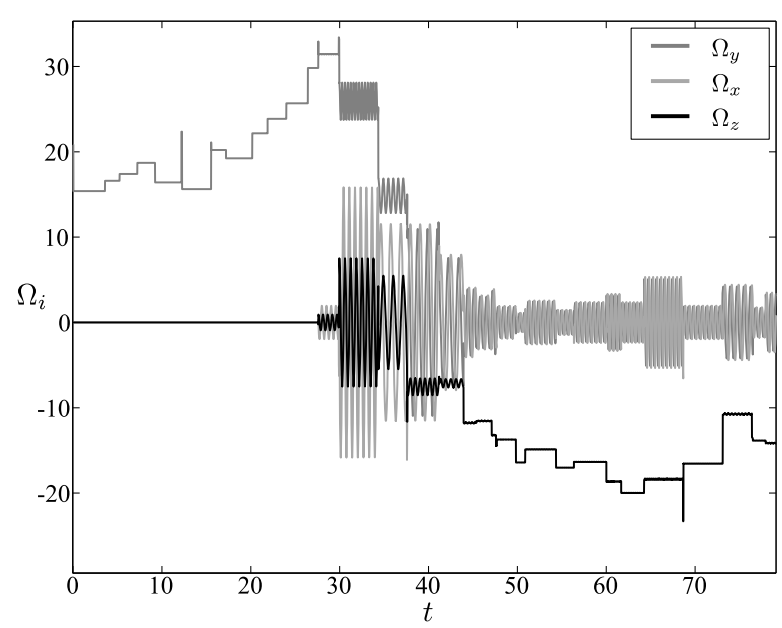

(b)

Fig. 12 (a) The block geometry. (b) Plot of the angular velocity components over time

excitation in the equation of motion [21]. The parametric resonances lead to highly complicated stability regions in the parameter space. The stabilization phenomenon in rockfall is therefore far from trivial. In this section, we will show that the stabilization phenomenon around the principal axis of largest inertia is present in the numerical simulation of a block on an inclined plane.

We consider a box shaped block with side lengths $l_{x}=2.5 \mathrm{~m}, l_{y}=3 \mathrm{~m}, l_{z}=1 \mathrm{~m}$ as depicted in Fig. 12(a). Furthermore, we assume that the block has a homogeneous mass distribution $\rho=3000 \mathrm{~kg} \mathrm{~m}^{-3}$. This results in a total mass $m=22,500 \mathrm{~kg}$ and the three distinct principal moments of inertia $\Theta_{x}=18,750 \mathrm{~kg} \mathrm{~m}^{2}, \Theta_{y}=13,594 \mathrm{~kg} \mathrm{~m}^{2}, \Theta_{z}=28,594 \mathrm{~kg} \mathrm{~m}^{2}$. The order $\Theta_{z}>\Theta_{x}>\Theta_{y}$ of the principal moments of inertia implies that the free body rotations about $\boldsymbol{e}_{y}^{B}$ and $\boldsymbol{e}_{z}^{B}$ are stable whereas rotation about $\boldsymbol{e}_{x}^{B}$ is unstable. The simulation domain consists of a plane with slope angle $27^{\circ}$. The coefficients of restitution for the Newton impact law are chosen completely inelastic (e.g. $\varepsilon_{N}=\varepsilon_{T}=0$ ). The friction coefficient is chosen quite high $\mu=10$ to ensure that the contacts never glide. The block is released slightly above the ground with an initial translational velocity $\left\|_{I} \boldsymbol{v}_{s}\right\|=28 \mathrm{~m} \mathrm{~s}^{-1}$ parallel to the slope and an initial angular velocity with the components $\Omega_{x}=\Omega_{z}=0$, $\Omega_{y}=20.8 \mathrm{rad} \mathrm{s}^{-1}$ in the body fixed frame. This corresponds to an angular velocity around the axis with the smallest principal moment of inertia $\Theta_{y}$.

The resulting development of the angular velocity components is plotted against time in Fig. 12(b). During the first 25 seconds, the block keeps rotating around $\boldsymbol{e}_{y}^{B}$ but at every impact tiny numerical errors in the generalized force directions of the contact forces and the contact percussions lead to $\Omega_{x}$ and $\Omega_{z}$ becoming nonzero causing a slight misalignment of the body to the slope. This misalignment eventually triggers an energy transfer into the other degrees of freedom. Finally, the body predominantly rotates around the $\boldsymbol{e}_{z}^{B}$ axis.

We conclude that the proposed simulation technique, which explicitly takes rock shape into account, is able to describe the observed wheel-like motion of tabular shaped rocks. This insight is important for rockfall research because it indicates that there are motions, which rocks can develop during run-out that result in straight and long run-outs, particularly for tabular and rounded shaped rocks. 


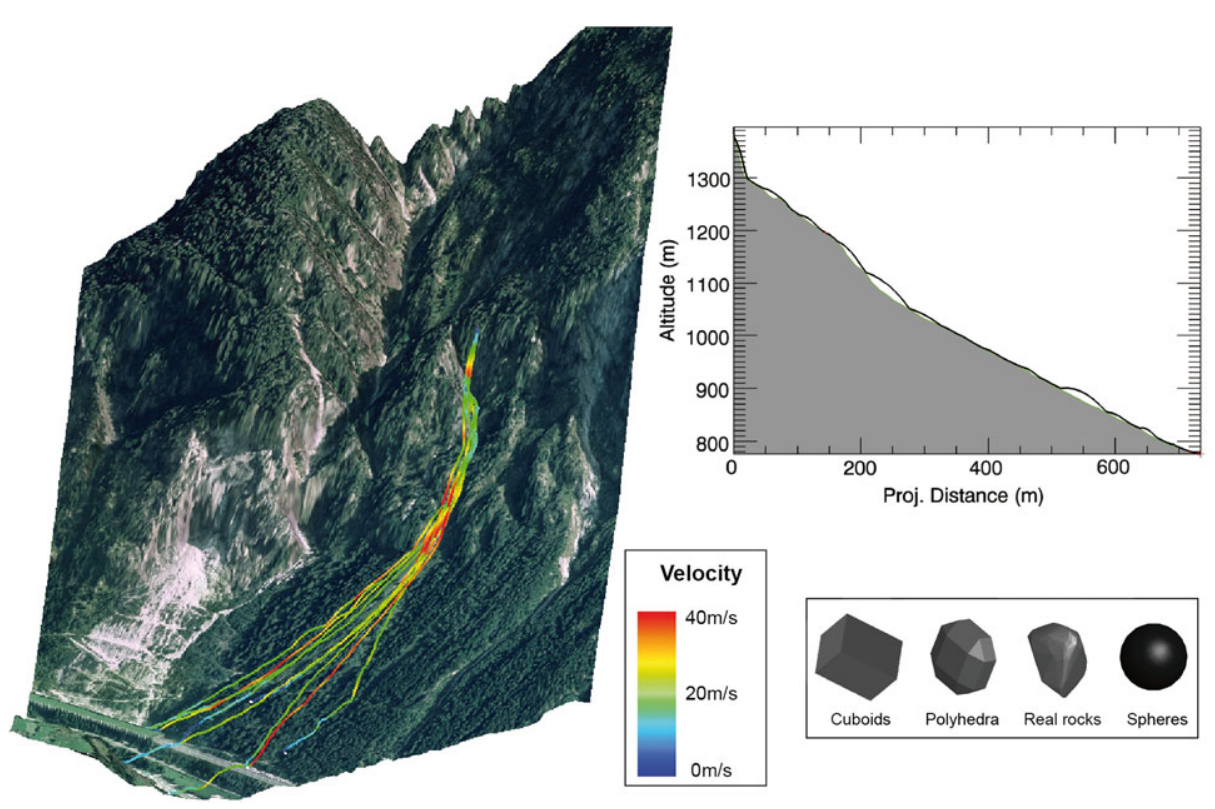

Fig. 13 Graphical user interface of the RAMMS rockfall module

\section{Conclusions}

In the present paper, state-of-the-art simulation techniques of nonsmooth contact dynamics and multibody dynamics have been applied to and adapted for the efficient simulation of rockfall trajectories. The presented method allows for the full three-dimensional simulation of rockfall events on arbitrary slopes and with arbitrary convex rock geometries. The geometry of the rock can be defined from point clouds, which are obtained from laser scans of real rocks in the field. The terrain geometry is constructed by bilinear interpolation of height values which are supplied on a regular uniform grid. The interaction of the rock with the terrain is modeled using hard unilateral constraints, which are numerically better tractable and allow for larger time-step sizes than the penalty approach. The time-integration scheme employed is energetically consistent to guarantee reliable energy information over the whole trajectory. The introduction of parameter fields for the friction coefficient $\mu$, the parameter of restitution $\varepsilon_{N}$ and other model parameters makes the model highly configurable and adaptable for the special needs of rockfall dynamics, where rocks encounter varied terrain properties over the run-out zone (rock, bush, meadow).

The simulation tool developed in this paper forms the core of the RAMMS rockfall module [8]. A screenshot of the graphical user interface is shown in Fig. 13. A variation in release position and orientation, as is observed from hazardous rock masses, allows for the statistical variability of rockfall trajectories required by engineers for rockfall hazard assessment [8].

The rock-forest interaction is modeled in the present paper by a simple drag force, which cannot describe the scattering effect caused by collisions with individual trees (sometimes referred to as the Galton box effect). However, a more elaborate forest model which includes cylindrical objects representing trees may seamlessly be incorporated in the presented nonsmooth contact dynamics approach. Further research is needed to develop a forest model, which accounts for the finite strength of trees. The RAMMS rockfall module, of which the 
simulation techniques are documented in this paper, is currently being tested and validated by rockfall experts through a comparison with field data.

The main merits of the paper for the research on rockfall dynamics are:

1. The influence of shape on the rolling behavior of bodies can be studied with the presented simulation method. As an example, the phenomenon of preferred rolling directions has been addressed in Sect. 5.2. The stabilization phenomenon around the principal axis of largest inertia causes a wheel-like behavior of the boulder and, therefore, greatly increases its run-out distance. The influence of rock shape is therefore crucial in rockfall dynamics and it is quintessential that rockfall simulation tools can properly reproduce dynamic phenomena induced by rock shape.

2. A novel friction model, which involves a slippage dependent friction coefficient, has been introduced in this paper. Using this friction model, it is possible to describe the scarring effect of rocks on the terrain, i.e., rocks tend to plough into the ground material, slide, and then lift off.

Although the paper has a clear focus on the application of rockfall, these merits may equally be of importance for other research fields. The gained insight on shape influence may be of importance for vibratory feeders and other conveyor systems for small parts, whereas the slippage dependent friction model may be used for the description of abrasive processes such as cutting processes in machine tool manufacturing.

Acknowledgements The project Multi-body Dynamics of Polygonised 3D Objects with Unilateral Frictional Contact: Application to Rockfall, being a cooperation between the SLF/WSL and the Center of Mechanics (ETH Zurich), has been funded by the Swiss National Science Foundation under project number 200021-119613/1.

\section{References}

1. Acary, V., Brogliato, B.: Numerical Methods for Nonsmooth Dynamical Systems; Applications in Mechanics and Electronics. Lecture Notes in Applied and Computational Mechanics., vol. 35. Springer, Berlin (2008)

2. Alart, P., Curnier, A.: A mixed formulation for frictional contact problems prone to Newton like solution methods. Comput. Methods Appl. Mech. Eng. 92, 353-375 (1991)

3. Andrew, R., Hume, H., Bartingale, R., Rock, A., Zhang, R.: CRSP-3D User's Manual Colorado Rockfall Simulation Program. Federal Highway Administration. Publication No. FHWA-CFL/TD-12-007 (2012)

4. Batista, M.: Steady motion of a rigid disk of finite thickness on a horizontal plane. Int. J. Non-Linear Mech. 41, 605-621 (2006)

5. Bourrier, F., Dorren, L., Nicot, F., Berger, F., Darve, F.: Toward objective rockfall trajectory simulation using a stochastic impact model. Geomorphology 110(3-4), 68-79 (2009)

6. Brogliato, B.: Nonsmooth Mechanics: Models, Dynamics and Control, 2nd edn. Communications and Control Engineering Series. Springer, London (1999)

7. Canudas de Wit, C., Olsson, H., Åström, K., Lischinsky, P.: A new model for control of systems with friction. IEEE Trans. Autom. Control 40(3), 419-425 (1995)

8. Christen, M., Bühler, Y., Bartelt, P., Leine, R., Glover, J., Schweizer, A., Graf, C., McArdell, B., Gerber, W., Deubelbeiss, Y., Feistl, T., Volkwein, A.: Integral hazard management using a unified software environment: numerical simulation tool "RAMMS" for gravitational natural hazards. In: Koboltschnig, G., Hübl, J., Braun, J. (eds.) Proceedings of 12th Congress INTERPRAEVENT, vol. 1, pp. 77-86 (2012)

9. Christen, M., Kowalski, J., Bartelt, P.: RAMMS: Numerical simulation of dense snow avalanches in three-dimensional terrain. Cold Reg. Sci. Technol. 63, 1-14 (2010)

10. Crosta, G., Agliardi, F.: Parametric evaluation of 3D dispersion of rockfall trajectories. Nat. Hazards Earth Syst. Sci. 4, 583-598 (2004)

11. Dimnet, E., Fremond, M., Gormaz, R., San Martin, J.: Collisions of rigid bodies, deformable bodies and fluids. In: Bathe, K. (ed.) Proceedings of 2nd MIT Conference on Computational Fluid and Solid Mechanics, Vols. 1 and 2, Amsterdam, 17-20 June, pp. 1317-1321. Elsevier/MIT Press, Cambridge (2003) 
12. Dorren, L.: A review of rockfall mechanics and modelling approaches. Prog. Phys. Geogr. 27(1), 69-87 (2003)

13. Dubois, F., Jean, M., Renouf, M., Mozul, R., Martin, A., Bagneris, M.: LMGC90. In: CSMA 2011 10e Colloque National en Calcul des Structures, May 2011. Presqu'île de Giens, Var (2011)

14. Frémond, M.: Rigid bodies collisions. Phys. Lett. A 204, 33-41 (1995)

15. Génot, F., Brogliato, B.: New results on Painlevé paradoxes. Eur. J. Mech. A, Solids 18, 653-677 (1999)

16. Giani, G.: Rock Slope Stability Analysis. Balkema, Rotterdam (1992)

17. Gilardi, G., Sharf, I.: Literature survey of contact dynamics modelling. Mech. Mach. Theory 37, 12131239 (2002)

18. Glocker, C.: Set-Valued Force Laws, Dynamics of Non-Smooth Systems. Lecture Notes in Applied Mechanics, vol. 1. Springer, Berlin (2001)

19. Glocker, C.: Energetic consistency conditions for standard impacts. Part I. Newton-type inequality impact laws and Kane's example. Multibody Syst. Dyn. 29, 77-117 (2013)

20. Glocker, C.: Energetic consistency conditions for standard impacts. Part II. Poisson-type inequality impact laws. Multibody Syst. Dyn. (2013). doi:10.1007/s11044-013-9387-2

21. Glukhikh, Y., Tkhai, V.: Parametric resonance in the problem of a heavy rigid body rolling along a straight line on a plane. Int. J. Appl. Math. Mech. 66(6), 971-984 (2002)

22. Guzzetti, F., Crosta, G., Detti, R., Agliardi, F.: STONE: a computer program for the three-dimensional simulation of rock-falls. Comput. Geosci. 28(9), 1079-1093 (2002)

23. Hunt, K.H., Crossley, F.R.E.: Coefficient of restitution interpreted as damping in vibroimpact. J. Appl. Mech. 42(2), 440-445 (1975)

24. Jean, M.: The non smooth contact dynamics method. Comput. Methods Appl. Mech. Eng. 177, 235-257 (1999)

25. Johnson, K.L.: Contact Mechanics. Cambridge University Press, Cambridge (1985)

26. Karnopp, D.: Computer simulation of stick-slip friction in mechanical dynamic systems. J. Dyn. Syst. Meas. Control 107, 100-103 (1985)

27. Kenner, R., Phillips, M., Danioth, C., Denier, C., Thee, P., Zgraggen, A.: Investigation of rock and ice loss in a recently deglaciated mountain rock wall using terrestrial laser scanning: Gemsstock, Swiss Alps. Cold Reg. Sci. Technol. 67(3), 157-164 (2011)

28. Lan, H., Martin, C.D., Lim, C.H.: RockFall analyst: a GIS extension for three-dimensional and spatially distributed rockfall hazard modeling. Comput. Geosci. 33(2), 262-279 (2007)

29. Lankarani, H.M., Nikravesh, P.E.: Continuous contact force models for impact analysis in multibody systems. Nonlinear Dyn. 5, 193-207 (1994)

30. Le Hir, C.: Forêt et chutes de blocs: méthodologie de modélisation spatialisée du rôle de protection. Ph.D. thesis, Cemagref/Université de Marne-La-Vallée (2005)

31. Le Hir, C., Dimnet, E., Berger, F., Dorren, L.: TIN-based 3D deterministic simulation of rockfall taking trees into account. Geophys. Res. Abstr. 8, 04157 (2006)

32. Leine, R.I., Brogliato, B., Nijmeijer, H.: Periodic motion and bifurcations induced by the Painlevé paradox. Eur. J. Mech. A, Solids 21(5), 869-896 (2002)

33. Leine, R.I., Nijmeijer, H.: Dynamics and Bifurcations of Non-Smooth Mechanical Systems. Lecture Notes in Applied and Computational Mechanics, vol. 18. Springer, Berlin (2004)

34. Leine, R.I., van Campen, D.H., de Kraker, A., van den Steen, L.: Stick-slip vibrations induced by alternate friction models. International Journal of Nonlinear Dynamics and Chaos in Engineering Systems 16(1), 41-54 (1998)

35. Leine, R.I., van de Wouw, N.: Stability and Convergence of Mechanical Systems with Unilateral Constraints. Lecture Notes in Applied and Computational Mechanics, vol. 36. Springer, Berlin (2008)

36. Möller, M.: Consistent integrators for nonsmooth dynamical systems. Ph.D. thesis, ETH, Zurich (2011)

37. Moreau, J.J.: Unilateral contact and dry friction in finite freedom dynamics. In: Moreau, J.J., Panagiotopoulos, P.D. (eds.) Non-smooth Mechanics and Applications. CISM Courses and Lectures, vol. 302, pp. 1-82. Springer, Wien (1988)

38. Nikravesh, P.E.: Computer-Aided Analysis of Mechanical Systems. Prentice-Hall, London (1988)

39. Rafiee, A., Vinches, M., Dubois, F.: The non-smooth contact dynamics method applied to the mechanical simulation of a jointed rock mass. In: Proceedings of the 7th EUROMECH Nonlinear Dynamics Conference (ENOC2011), Rome (2011)

40. Studer, C.: Numerics of Unilateral Contacts and Friction-Modeling and Numerical Time Integration in Non-smooth Dynamics. Lecture Notes in Applied and Computational Mechanics, vol. 47. Springer, Heidelberg (2009)

41. Studer, C., Leine, R.I., Glocker, C.: Step size adjustment and extrapolation for time stepping schemes in nonsmooth dynamics. Int. J. Numer. Methods Eng. 76(11), 1747-1781 (2008) 
42. Volkwein, A., Schellenberg, K., Labiouse, V., Agliardi, F., Berger, F., Bourrier, F., Dorren, L.K.A., Gerber, W., Jaboyedoff, M.: Rockfall characterisation and structural protection-a review. Nat. Hazards Earth Syst. Sci. 11(9), 2617-2651 (2011)

43. Woltjer, M., Rammer, W., Brauner, M., Seidl, R., Mohren, G.M.J., Lexer, M.J.: Coupling a 3D patch model and a rockfall module to assess rockfall protection in mountain forests. J. Environ. Manag. 87(3), 373-388 (2008) 